\title{
Control of the supercurrent in a mesoscopic four-terminal Josephson junction
}

\author{
Qing-feng Sun \\ Department of Physics, The University of Hong Kong, Pokfulam Road, Hong Kong, China \\ and Department of Physics, State Key Laboratory for Mesoscopic Physics, Peking University, 100871 Beijing, China \\ Jian Wang \\ Department of Physics, The University of Hong Kong, Pokfulam Road, Hong Kong, China \\ Tsung-han Lin \\ Department of Physics, The University of Hong Kong, Pokfulam Road, Hong Kong, China \\ and Department of Physics, State Key Laboratory for Mesoscopic Physics, Peking University, 100871 Beijing, China
}

(Received 29 November 1999)

\begin{abstract}
We study the control of the supercurrent in a mesoscopic four-terminal superconductor-normal-metalsuperconductor (SNS) junction, in which the $N$ region is a quantum dot connected via tunneling barriers to two superconducting electrodes and two normal electrodes, respectively. By using the nonequilibrium Green's function method, the current flowing into the quantum dot from each electrode is derived. We find that the supercurrent between two superconducting electrodes can be suppressed and even reversed by changing the dc voltage applied across the two normal terminals, similar to recent experiments of diffusive SNS junctions and previous theories for both the ballistic and diffusive SNS junctions. Then we investigate a three-terminal SNS junction, reduced from the four-terminal junction by decoupling the dot from one normal terminal. We find that even at zero bias of the normal terminal, the supercurrent still can be controlled by changing the coupling strength between the dot and the normal terminal. In addition, both the Andreev reflection current and Andreev quasibound states depend on the phase difference of two superconductors and the coupling strength between the dot and superconducting electrodes. Finally, the behavior of the supercurrent is discussed in the limit when the normal terminals are decoupled from the system.
\end{abstract}

\section{INTRODUCTION}

One of the most intriguing experimental results on mesoscopic superconductivity has been controlling the supercurrent through a superconductor-normal-metal-superconductor (SNS) Josephson junction by an external voltage applied across the normal metal of the structure, not only because of fundamental interest, but also of potential applications for future nanoelectronics. ${ }^{1}$

The earliest work on this subject was limited on theoretical studies, either for ballistic SNS junction, ${ }^{2-4}$ or for diffusive SNS junction..$^{5-7}$ In ballistic SNS junction, since the elastic mean free path of quasiparticles in normal region $(N$ region) is longer than the length of it, Andreev bound states can be formed, ${ }^{8}$ which have such feature that each successive Andreev bound state carries a supercurrent in opposite direction at a given phase difference $\phi$ between the two superconductors. Therefore, the net supercurrent between two superconductors depends not only on the phase difference $\phi$, but also on the occupation of the Andreev quasibound states. Authors in Refs. 2-4 predicted theoretically that the supercurrent may be modulated by changing the distribution of quasiparticles in the $N$ region. For diffusive SNS junction, the concept of Andreev bound states is not appropriate since the electron trajectories are not well defined. Instead, the so-called supercurrent-carrying density of states, an analogue of Andreev bound state, has the positive and the negative parts at a given phase difference and plays the similar role. ${ }^{1}$ Theories ${ }^{5-7}$ predicted that the magnitude and direction of the total supercurrent depend on the occupied fraction of these states, similar to the occupation of the discrete Andreev bound states in the ballistic SNS junctions.

The first experiment manifesting the control of the supercurrent was performed by Morpurgo et al. ${ }^{9}$ for a diffusive SNS junction. They demonstrated that the supercurrent between two superconducting electrodes can be modulated by changing the dc voltage across the $N$ region of the structure. In order to explain their experimental results, they proposed a theoretical model based on a quasiequilibrium distribution with locally enhanced effective electron temperature. The observation of a monotonic decrease of the supercurrent in their experiment is consistent with their model.

Lately, Wilhelm et al. ${ }^{10}$ studied theoretically a mesoscopic diffusive SNS junction. Different from the situation of the experiment by Morpurgo et al., they assumed that the inelastic scattering in the $N$ region can be neglected. By using the quasiclassical Keldysh Green's function approach, they showed that the distribution of quasiparticles in the mesoscopic $N$ region can be driven to a nonequilibrium twostep function form by a dc voltage across the $N$ region, as observed in the experiment by Pothier et al. ${ }^{11}$ They attributed the suppression of the supercurrent to the nonequilibrium quasiparticle distribution in the $N$ region, and predicted the possibility of a transition to a $\pi$ junction.

Very recently, Baselmans et al. ${ }^{1}$ successfully demonstrated the suppression of the supercurrent in a controllable Josephson junction. The setup used in their experiment is a mesoscopic diffusive SNS junction in which the normal 


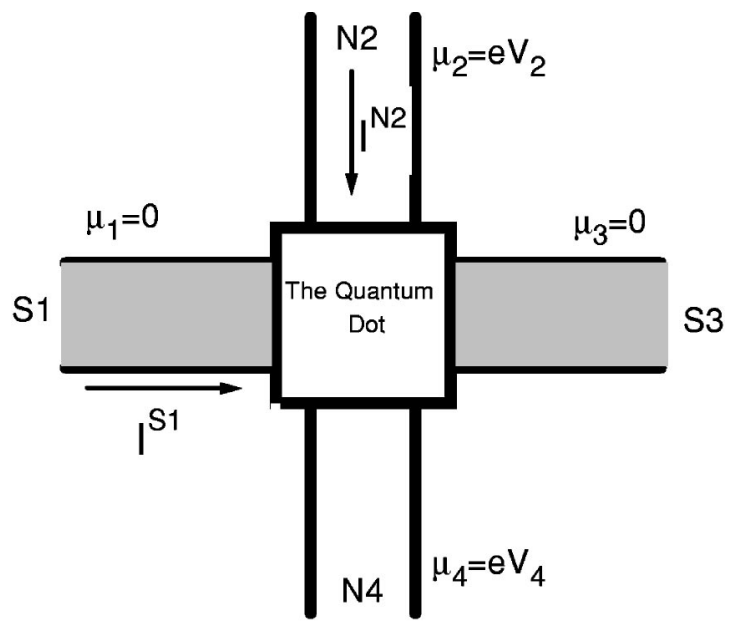

FIG. 1. A Schematic diagram for the four-terminal SNS junction: the $N$ region is a quantum dot (with a single energy level), connected to two superconducting terminals $S 1$ and $S 3$ and two normal terminals $N 2$ and $N 4$, respectively.

metal is a gold wire connected to two normal reservoirs. The controlling dc voltage across the gold wire induces a position-dependent nonequilibrium quasiparticle distribution in the $N$ region, resulting in a modulation of the supercurrent. The experiment also manifested a reversal of the direction of the supercurrent under a certain value of the dc voltage, i.e., a transition to the $\pi$ junction.

In this work we consider a mesoscopic four-terminal SNS junction (see Fig. 1) in which the $N$ region is a quantum dot, connected via tunneling barriers to two superconducting electrodes ( $S 1$ and $S 3$ ) and two normal electrodes ( $N 2$ and N4), respectively. The motivation of this work is to check if it is still possible to have the control of the supercurrent in this mesoscopic four-terminal SNS junction. Different from both the ballistic and diffusive SNS junctions studied before, the transport through a quantum dot with discrete energy levels is typically in resonant tunneling regime, and the suppression of the supercurrent for such a SNS junction has not been studied yet. By using the nonequilibrium Green's function (NGF) method, we investigate in detail different types of the current, including the supercurrent and normal current, flowing into the central region (the quantum dot) from the superconducting and the normal terminals. Based on the current formula we obtained, we first study numerically the four-terminal SNS junction, with the superconducting electrodes at zero voltage [i.e., $V_{1}=V_{3}=0$, where $V_{n} \quad(n=1,2$, 3 , and 4 ) is the dc voltage of the $n$th terminal], and the normal electrodes at equal and opposite voltages $\left(V_{2}=\right.$ $-V_{4} \equiv V / 2$, where $V$ is the dc bias voltage). We find the following. (1) The suppression of the magnitude of the supercurrent still exists by tuning the dc bias voltage $V$ across the $N$ region. In addition, when the dc bias $V$ exceeds a critical value $V_{c}$, the supercurrent will reverse its direction, i.e., a transition to a $\pi$ junction will occur, due to the change of electron distribution in the quantum dot. (2) The current from the normal terminal $N 2, I^{N 2}$, is the normal current, which can be separated into two parts for $V / 2<\Delta \quad$ ( $\Delta$ is the superconducting energy gap), $I_{A}^{N 2}$ and $I_{c}^{N 2}: I_{A}^{N 2}$ originates from the Andreev reflection and approximately has the current-phase relation as $I_{A}^{N 2}(\phi)=I_{A}^{N 2}(0)(1+\cos \phi) / 2$, where $\phi$ is the phase difference of two superconducting terminal; $I_{c}^{N 2}$ results from the conventional electron tunneling and is approximately $I_{c}^{N 2}(\phi)=I_{c}^{N 2}(0)(1+\alpha \cos \phi) /(1+\alpha)$ $(-1<\alpha<0)$. These two parts of the normal current have different forms of the phase dependence, leading to a complicated current-phase relation. Second, we study the threeterminal case simply by decoupling the SNS junction from the normal terminal $N 4$. When voltages of all three terminals are set to be zero $\left(V_{1}=V_{2}=V_{3}=0\right)$, only the supercurrent may still flow between two superconducting leads if the phase difference is nonzero. We find that the supercurrent can still be controlled by changing the coupling strength between the dot and the normal lead $N 2, \Gamma_{2}$, even if no current flows from $N 2$ into the dot and the distribution of electrons in the dot remains unchanged. While $V_{2} \neq 0$, both the supercurrent and normal current depend on the coupling strength $\Gamma_{2}$ and on the phase difference $\phi$; and the Andreev quasibound states play a crucial role. Finally, in the limit of $\Gamma_{2} \rightarrow 0$, we find that the supercurrent still depends on the voltage of the normal terminal $N 2, V_{2}$, as obtained before by Wees et al. by using Bogoliubov-de Gennes equation and the scattering matrix method. ${ }^{2}$

The rest of this paper is organized as follows. In Sec. II we present the model and derive the current formula $I^{S 1}$ (flowing into the dot from $S 1$ ) and $I^{N 2}$ (flowing into the dot from N2) by using the NGF technique. In Sec. III we present the numerical studies for the four-terminal case, including the suppression and reversal of the supercurrent by tuning the dc bias voltage between two normal terminals $N 2$ and $N 4$; and the behavior of the normal current. The threeterminal case is investigated in Sec. IV, including the dependence of the supercurrent, the Andreev reflection current, and the Andreev quasibound states on the phase difference $\phi$ and the coupling strength $\Gamma_{2}$. In Sec. $V$ the behavior of the currents in the limit of $\Gamma_{2} \rightarrow 0$ is discussed. Finally, a brief summary is given in Sec. VI.

\section{MODEL AND FORMULATION}

We assume that the system under consideration (see Fig. $1)$ is described by the following Hamiltonian: ${ }^{12-15}$

$$
H=\sum_{n=1,2,3,4} H_{\text {lead- } n}+H_{\text {dot }}+H_{T},
$$

with

$$
\begin{gathered}
H_{\text {lead- } n}=\sum_{k \sigma} \epsilon_{n k} a_{n, k \sigma}^{\dagger} a_{n, k \sigma}+\sum_{k}\left[\Delta_{n} a_{n, k \downarrow} a_{n,-k \uparrow}\right. \\
\left.+\Delta_{n}^{*} a_{n,-k \uparrow}^{\dagger} a_{n, k \downarrow}^{\dagger}\right], \\
H_{\mathrm{dot}}=\sum_{\sigma} \epsilon_{0} c_{\sigma}^{\dagger} c_{\sigma}, \\
H_{T}=\sum_{n, k, \sigma}\left[v_{n k} a_{n, k \sigma}^{\dagger} c_{\sigma}+v_{n k}^{*} c_{\sigma}^{\dagger} a_{n, k \sigma}\right],
\end{gathered}
$$

where $H_{\text {lead- } n}$ describes electrons in the $n$th lead, $a_{n, k \sigma}^{\dagger}\left(a_{n, k \sigma}\right)$ are the creation (annihilation) operators of the electron, $\Delta_{n}=\left|\Delta_{n}\right| e^{i \phi_{n}}$ is the complex superconducting order 
parameter of the $n$th lead, with $\left|\Delta_{n}\right|$ the superconducting energy gap, and $\phi_{n}$ the superconducting phase. ${ }^{16}$ Since we have assumed that terminals $N 2$ and $N 4$ are normal leads, so $\Delta_{2}=\Delta_{4}=0$. In this work we will set voltages of the superconducting terminals to be zero, i.e., $V_{1}=V_{3}=0$ where $V_{n}$ is the voltage of the $n$th terminal. Here $H_{\text {dot }}$ is the Hamiltonian of the central $N$ region, which we assume is a quantum dot with a single energy level. For simplicity, the intradot electron-electron Coulomb interaction is not considered, namely, we only consider a big dot. $H_{T}$ denotes the tunneling part of the Hamiltonian, with $v_{n k}$ being the hopping matrix element.

To facilitate the calculation, we perform a transformation of the Hamiltonian using the unitary operator $U$ $=\exp \left[\sum_{n, k, \sigma}\left(i \phi_{n} / 2 \hbar\right) a_{n, k \sigma}^{\dagger} a_{n, k \sigma}\right] .{ }^{17}$ Then, $H_{\text {lead- } n}$ and $H_{T}$ become

$$
\begin{aligned}
H_{\text {lead- } n}= & \sum_{k, \sigma} \epsilon_{n k} a_{n, k \sigma}^{\dagger} a_{n, k \sigma}+\sum_{k}\left[\left|\Delta_{n}\right| a_{n, k \downarrow} a_{n,-k \uparrow}\right. \\
& \left.+\left|\Delta_{n}\right| a_{n,-k \uparrow}^{\dagger} a_{n, k \downarrow}^{\dagger}\right], \\
H_{T} & =\sum_{n, k, \sigma}\left\{v_{n k} e^{i \phi_{n} / 2 \hbar} a_{n, k \sigma}^{\dagger} c_{\sigma}+\text { H.c. }\right],
\end{aligned}
$$

and $H_{\text {dot }}$ is unchanged. Hereafter, we will assume $\left|\Delta_{1}\right|$ $=\left|\Delta_{3}\right| \equiv \Delta$ for simplicity.

The current flowing into the dot from the $n$th terminal can be calculated from the evolution of the total number operator of the electrons in the $\operatorname{dot} N_{n}=\sum_{k, \sigma} a_{n, k \sigma}^{\dagger} a_{n, k \sigma}$ (in units of $\hbar=1)::^{18,19}$

$$
\begin{aligned}
I^{S(N) n}(t) & =-e\left\langle\dot{N}_{n}(t)\right\rangle=i e\left\langle\left[N_{n}, H\right]\right\rangle \\
& =4 e \operatorname{Re} \sum_{k} v_{n k} e^{i \phi_{n} / 2} G_{n k ; 11}^{<}(t, t),
\end{aligned}
$$

where $I^{S n}$ is for $n=1$ or 3 , and $I^{N n}$ for $n=2$ or $4 ; \mathbf{G}_{n k}^{<}(t, t)$ is the matrix Green function in $2 \times 2$ Nambu representation, defined by ${ }^{12,20}$

$$
\mathbf{G}_{n k}^{<}\left(t, t^{\prime}\right) \equiv i\left(\begin{array}{ll}
\left\langle a_{n, k \uparrow}^{\dagger}\left(t^{\prime}\right) c_{\uparrow}(t)\right\rangle & \left\langle a_{n, k \downarrow}\left(t^{\prime}\right) c_{\uparrow}(t)\right\rangle \\
\left\langle a_{n, k \uparrow}^{\dagger}\left(t^{\prime}\right) c_{\downarrow}^{\dagger}(t)\right\rangle & \left\langle a_{n, k \downarrow}\left(t^{\prime}\right) c_{\downarrow}^{\dagger}(t)\right\rangle
\end{array}\right) .
$$

Notice that we only need to derive the expressions of the current $I^{S 1}$ and $I^{N 2}$; while the current $I^{S 3}$ and $I^{N 4}$ can be easily obtained from $I^{S 1}$ and $I^{N 2}$ by exchanging the terminal index 1 and 3, 2, and 4, respectively.

The current $I^{S 1}$ can be expressed in terms of the dot's Green functions $\mathbf{G}^{r}$ and $\mathbf{G}^{<}$, defined in Nambu representation by ${ }^{14,21}$

$$
\begin{aligned}
& \mathbf{G}^{r}\left(t, t^{\prime}\right) \equiv-i \theta\left(t-t^{\prime}\right) \\
& \times\left(\begin{array}{ll}
\left\langle\left\{c_{\uparrow}(t), c_{\uparrow}^{\dagger}\left(t^{\prime}\right)\right\}\right\rangle & \left\langle\left\{c_{\uparrow}(t), c_{\downarrow}\left(t^{\prime}\right)\right\}\right\rangle \\
\left\langle\left\{c_{\downarrow}^{\dagger}(t), c_{\uparrow}^{\dagger}\left(t^{\prime}\right)\right\}\right\rangle & \left\langle\left\{c_{\downarrow}^{\dagger}(t), c_{\downarrow}\left(t^{\prime}\right)\right\}\right\rangle
\end{array}\right), \\
& \mathbf{G}^{<}\left(t, t^{\prime}\right) \equiv i\left(\begin{array}{ll}
\left\langle c_{\uparrow}^{\dagger}\left(t^{\prime}\right) c_{\uparrow}(t)\right\rangle & \left\langle c_{\downarrow}\left(t^{\prime}\right) c_{\uparrow}(t)\right\rangle \\
\left\langle c_{\uparrow}^{\dagger}\left(t^{\prime}\right) c_{\downarrow}^{\dagger}(t)\right\rangle & \left\langle c_{\downarrow}\left(t^{\prime}\right) c_{\downarrow}^{\dagger}(t)\right\rangle
\end{array}\right) .
\end{aligned}
$$

Following the same procedure as in Ref. 19 (see Appendixes $\mathrm{A}$ and $\mathrm{B}$ there), one has

$$
\begin{aligned}
\mathbf{G}_{n k}^{<}(t, t)= & \int d t_{1}\left[\mathbf{G}^{r}\left(t, t_{1}\right) \mathbf{v}_{n k}^{*} \mathbf{g}_{n k, n k}^{<}\left(t_{1}, t\right)\right. \\
& \left.+\mathbf{G}^{<}\left(t, t_{1}\right) \mathbf{v}_{n k}^{*} \mathbf{g}_{n k, n k}^{a}\left(t_{1}, t\right)\right],
\end{aligned}
$$

where $\mathbf{v}_{n k}$ is a $2 \times 2$ matrix of the hopping elements defined by

$$
\mathbf{v}_{n k}=\left(\begin{array}{ll}
v_{n k} e^{i \phi_{n} / 2} & 0 \\
0 & -v_{n k}^{*} e^{-i \phi_{n} / 2}
\end{array}\right),
$$

and $\mathbf{g}_{n k, n k}^{<, a}\left(t_{1}, t\right)$ are the exact Green functions of the $n$th terminal without the coupling to the dot. Assuming the normal density of states $\rho_{1}^{N}(\epsilon)$ is independent of $\epsilon$, one has $^{12,14,21}$

$\sum_{k} \mathbf{g}_{1 k, 1 k}^{<}\left(t_{1}, t\right)$

$$
=i \int d \epsilon \rho_{1}^{N} f_{1}(\epsilon) \tilde{\rho}(\epsilon) e^{-i \epsilon\left(t_{1}-t\right)}\left(\begin{array}{ll}
1 & \Delta / \epsilon \\
\Delta / \epsilon & 1
\end{array}\right),
$$

$\sum_{k} \mathbf{g}_{1 k, 1 k}^{a}\left(t_{1}, t\right)$

$$
=i \theta\left(t-t_{1}\right) \int d \epsilon \rho_{1}^{N} \beta^{*}(\epsilon) e^{-i \epsilon\left(t_{1}-t\right)}\left(\begin{array}{ll}
1 & \Delta / \epsilon \\
\Delta / \epsilon & 1
\end{array}\right),
$$

where $\beta^{*}(\epsilon)$ is the complex conjugate of $\beta(\epsilon)$, with $\beta(\epsilon)$ $=\epsilon / i \sqrt{\Delta^{2}-\epsilon^{2}}$ for $\Delta>|\epsilon|$ and $\beta(\epsilon)=|\epsilon| / \sqrt{\epsilon^{2}-\Delta^{2}}$ for $\Delta$ $<|\epsilon|$. $\tilde{\rho}(\epsilon)=\operatorname{Re}[\beta(\epsilon)]=\theta(|\epsilon|-\Delta)|\epsilon| / \sqrt{\epsilon^{2}-\Delta^{2}}$ is the dimensionless BCS density of states, i.e., the ratio of the superconducting density of states $\rho^{S}(\epsilon)$ to the normal density of states $\rho^{N}(\epsilon)$. Function $f_{1}(\epsilon)$ in Eq. (10) is the Fermi distribution of the electrons: $f_{1}(\epsilon)=\left[\exp \left(\epsilon / k_{B} \mathcal{T}\right)+1\right]^{-1}$, in which $\mathcal{T}$ is the temperature.

Substituting the Green functions $\mathbf{g}_{1 k, 1 k}^{<, a}$ and hopping matrix $\mathbf{v}_{1 k}$ into Eq. (9), we obtain

$$
\begin{aligned}
G_{1 k ; 11}^{<}(t, t)= & \int d t_{1}\left\{G_{11}^{r}\left(t, t_{1}\right) v_{1 k ; 11}^{*} g_{1 k, 1 k ; 11}^{<}\left(t_{1}, t\right)\right. \\
& +G_{12}^{r}\left(t, t_{1}\right) v_{1 k ; 22}^{*} g_{1 k, 1 k ; 21}^{<}\left(t_{1}, t\right) \\
& +G_{11}^{<}\left(t, t_{1}\right) v_{1 k ; 11}^{*} g_{1 k, 1 k ; 11}^{a}\left(t_{1}, t\right) \\
& \left.+G_{12}^{<}\left(t, t_{1}\right) v_{1 k ; 22}^{*} g_{1 k, 1 k ; 21}^{a}\left(t_{1}, t\right)\right\},
\end{aligned}
$$

in which $G_{i j}^{r,<}(i, j=1,2)$ are the matrix elements of $\mathbf{G}^{r,<}$. Substituting $G_{1 k ; 11}^{<}(t, t)$ into Eq. (5), assuming $v_{n k}$ is real and independent of index $k,{ }^{22}$ the current $I^{S 1}$ can be expressed in terms of the dot's Green functions as

$$
\begin{aligned}
I^{S 1}= & -4 e I m \int \frac{d \epsilon}{2 \pi} \Gamma_{1}(\epsilon) \\
& \times\left\{\left[f_{1}(\epsilon) \tilde{\rho}(\epsilon) G_{11}^{r}(\epsilon)+\frac{1}{2} \beta^{*}(\epsilon) G_{11}^{<}(\epsilon)\right]\right. \\
& \left.-\frac{\Delta}{\epsilon} e^{i \phi_{1}}\left[f_{1}(\epsilon) \tilde{\rho}(\epsilon) G_{12}^{r}(\epsilon)+\frac{1}{2} \beta^{*}(\epsilon) G_{12}^{<}(\epsilon)\right]\right\},
\end{aligned}
$$


where $\Gamma_{n}(n=1,2,3$, and 4$)$ is the linewidth function defined by $\Gamma_{n}(\epsilon) \equiv 2 \pi v_{n}^{2} \rho_{n}^{N}(\epsilon)$, which represents the coupling strength between the dot and $n$th lead; $G_{i j}^{r,<}(\epsilon)$ are the Fourier transformation of $G_{i j}^{r,<}\left(t, t_{1}\right)$. For the situation of $V_{1}$ $=V_{3}=0, G_{i j}^{r,<}\left(t, t_{1}\right)$ depends only on the time difference $\left(t-t_{1}\right)$, which has been used in derivation of Eq. (14). Notice that the anomalous Green function $G_{12}^{r}$ and $G_{12}^{<}$emerge in the current formula Eq. (14), indicating the presence of the superconducting leads and leading to the Josephson supercurrent. It should be mentioned that Eq. (14) can also be obtained directly from a general formula obtained recently by Sun et al. for a mesoscopic hybrid multi-terminal system. ${ }^{17}$ That general formula can be used to describe the case with arbitrarily finite voltage at each terminal, any type of interactions in the central region, and any portion of the system subjected to external microwave fields.

The current flowing into the dot from normal terminal $N 2$ can be easily expressed as ${ }^{18,19}$

$$
I^{N 2}=-4 e \operatorname{Im} \int \frac{d \epsilon}{2 \pi} \Gamma_{2}(\epsilon)\left\{f_{2}(\epsilon) G_{11}^{r}(\epsilon)+\frac{1}{2} G_{11}^{<}(\epsilon)\right\},
$$

where $f_{2}(\epsilon)$ is the Fermi distribution function of electrons in normal terminal $N 2, f_{2}(\epsilon)=\left\{\exp \left[\left(\epsilon-e V_{2}\right) / k_{B} \mathcal{T}\right]+1\right\}^{-1}$. Notice that the expression of $I^{N 2}$, Eq. (15), holds for any value of $V_{2}$, but the expression of $I^{S 1}$, Eq. (14), is valid only for $V_{1}=V_{3}=0$.

Now we need to solve for the Green functions $\mathbf{G}^{r}(\boldsymbol{\epsilon})$ and $\mathbf{G}^{<}(\boldsymbol{\epsilon})$ of the dot. First, $\mathbf{G}^{r}(\boldsymbol{\epsilon})$ can be solved from Dyson's equation

$$
\mathbf{G}^{r}=\mathbf{g}^{r}+\mathbf{G}^{r} \mathbf{\Sigma}^{r} \mathbf{g}^{r}
$$

in which we have dropped the argument $\epsilon . \boldsymbol{\Sigma}^{r}(\epsilon)$ is the self-energy and $\mathbf{g}^{r}(\boldsymbol{\epsilon})$ is the dot's Green function without the coupling between the dot and four leads. By taking the widebandwidth approximation, the linewidth $\Gamma_{n}(\epsilon)$ becomes independent of the energy $\epsilon{ }^{21}$ Notice that $\Gamma_{n}=2 \pi v_{n}^{2} \rho_{n}^{N}(\epsilon)$, where $\rho_{n}^{N}(\epsilon)$ is the density of states of the $n$th terminal lead in normal state, so here the wide-bandwidth approximation is generally reasonable as in the case of the normal system. ${ }^{23}$ Under the wide-bandwidth approximation, the self-energy $\boldsymbol{\Sigma}^{r}(\boldsymbol{\epsilon})$ can be written as (see Ref. 21)

$$
\boldsymbol{\Sigma}^{r}(\boldsymbol{\epsilon})=\sum_{n=1,2,3,4} \boldsymbol{\Sigma}_{n}^{r}(\boldsymbol{\epsilon})
$$

For the superconducting terminal $n=1$ or $3, \boldsymbol{\Sigma}_{n}^{r}(\epsilon)$ has the form

$$
\begin{aligned}
\boldsymbol{\Sigma}_{n}^{r}(\boldsymbol{\epsilon})= & \sum_{k} \mathbf{v}_{n} \mathbf{g}_{n k, n k}^{r}(\boldsymbol{\epsilon}) \mathbf{v}_{n}^{*}=-\frac{i \Gamma_{n}}{2} \beta(\boldsymbol{\epsilon}) \\
& \times\left(\begin{array}{cc}
1 & -\frac{\Delta}{\epsilon} e^{-i \phi_{n}} \\
-\frac{\Delta}{\epsilon} e^{i \phi_{n}} & 1
\end{array}\right),
\end{aligned}
$$

while for the normal terminal $n=2$ or $4, \Sigma_{n}^{r}(\boldsymbol{\epsilon})$ is

$$
\boldsymbol{\Sigma}_{n}^{r}(\boldsymbol{\epsilon})=\sum_{k} \mathbf{v}_{n} \mathbf{g}_{n k, n k}^{r}(\boldsymbol{\epsilon}) \mathbf{v}_{n}^{*}=-\frac{i \Gamma_{n}}{2}\left(\begin{array}{ll}
1 & 0 \\
0 & 1
\end{array}\right) .
$$

The Green function $\mathbf{g}^{r}(\epsilon)$ can be easily obtained as

$$
\mathbf{g}^{r}(\boldsymbol{\epsilon})=\left(\begin{array}{ll}
\left(\boldsymbol{\epsilon}-\boldsymbol{\epsilon}_{0}+i 0^{+}\right)^{-1} & 0 \\
0 & \left(\boldsymbol{\epsilon}+\boldsymbol{\epsilon}_{0}+i 0^{+}\right)^{-1}
\end{array}\right) .
$$

With the help of $\boldsymbol{\Sigma}^{r}(\boldsymbol{\epsilon})$ and $\mathbf{g}^{r}(\boldsymbol{\epsilon}), \mathbf{G}^{r}(\boldsymbol{\epsilon})$ can be solved exactly from Dyson's equation Eq. (16), as

$$
\mathbf{G}^{r}(\boldsymbol{\epsilon})=A\left(\begin{array}{ll}
\left(g_{22}^{r-1}-\Sigma_{22}^{r}\right) & \Sigma_{12}^{r} \\
\Sigma_{21}^{r} & \left(g_{11}^{r-1}-\Sigma_{11}^{r}\right)
\end{array}\right),
$$

where $A(\epsilon)$ is a compact notation defined by

$$
A=\left[\left(g_{11}^{r-1}-\Sigma_{11}^{r}\right)\left(g_{22}^{r-1}-\Sigma_{22}^{r}\right)-\Sigma_{12}^{r} \Sigma_{21}^{r}\right]^{-1},
$$

in which $g_{i j}^{r}$ or $\sum_{i j}^{r}(i, j=1,2)$ is the matrix element of the $2 \times 2$ matrix $\mathbf{g}^{r}$ or $\boldsymbol{\Sigma}^{r}$.

Second, we solve the lesser Green function $G_{11}^{<}(\epsilon)$ and $G_{12}^{<}(\boldsymbol{\epsilon})$. From Keldysh equation, $\mathbf{G}^{<}=\mathbf{G}^{r} \boldsymbol{\Sigma}^{<} \mathbf{G}^{a}$, one has

$$
\begin{aligned}
& G_{11}^{<}=G_{11}^{r} \Sigma_{11}^{<} G_{11}^{a}+G_{11}^{r} \Sigma_{12}^{<} G_{21}^{a}+G_{12}^{r} \Sigma_{21}^{<} G_{11}^{a}+G_{12}^{r} \Sigma_{22}^{<} G_{21}^{a}, \\
& G_{12}^{<}=G_{11}^{r} \Sigma_{11}^{<} G_{12}^{a}+G_{11}^{r} \Sigma_{12}^{<} G_{22}^{a}+G_{12}^{r} \Sigma_{21}^{<} G_{12}^{a}+G_{12}^{r} \Sigma_{22}^{<} G_{22}^{a},
\end{aligned}
$$

where $\mathbf{G}^{a}(\boldsymbol{\epsilon})=\left[\mathbf{G}^{r}(\boldsymbol{\epsilon})\right]^{*}$ and $\boldsymbol{\Sigma}^{<}(\boldsymbol{\epsilon})$ is the lesser self-energy. Under the wide-bandwidth approximation, $\boldsymbol{\Sigma}^{<}(\boldsymbol{\epsilon})$ has the following form: ${ }^{21}$

$$
\mathbf{\Sigma}^{<}(\boldsymbol{\epsilon})=\sum_{n=1,2,3,4} \mathbf{\Sigma}_{n}^{<}(\boldsymbol{\epsilon})
$$

For the superconducting terminal $\left(n=1\right.$ or 3 , with $V_{1}=V_{3}$ $=0$ )

$$
\boldsymbol{\Sigma}_{n}^{<}(\boldsymbol{\epsilon})=i \Gamma_{n} f_{n}(\boldsymbol{\epsilon}) \tilde{\rho}(\boldsymbol{\epsilon})\left(\begin{array}{ll}
1 & -\frac{\Delta}{\epsilon} e^{-i \phi_{n}} \\
-\frac{\Delta}{\epsilon} e^{i \phi_{n}} & 1
\end{array}\right)
$$

while for the normal terminal $(n=2$ or 4$)$

$$
\boldsymbol{\Sigma}_{n}^{<}(\boldsymbol{\epsilon})=i \Gamma_{n}\left(\begin{array}{ll}
f_{n}(\boldsymbol{\epsilon}) & 0 \\
0 & \tilde{f}_{n}(\boldsymbol{\epsilon})
\end{array}\right)
$$

where $\widetilde{f}_{n}(\epsilon)$ is the Fermi distribution function of holes in the $n$th terminal: $\widetilde{f}_{n}(\epsilon)=1-f_{n}(-\epsilon)$. Substituting the selfenergy $\boldsymbol{\Sigma}^{<}(\boldsymbol{\epsilon})$, Eqs. (25)-(27), into Keldysh equation, the Green functions $G_{11}^{<}(\epsilon)$ and $G_{12}^{<}(\epsilon)$ are obtained as

$$
\begin{aligned}
G_{11}^{<}= & i\left|G_{11}^{r}\right|^{2}\left[\Gamma_{1} \tilde{\rho} f_{1}+\Gamma_{3} \tilde{\rho} f_{3}+\Gamma_{2} f_{2}+\Gamma_{4} f_{4}\right] \\
& -i G_{11}^{r} G_{12}^{r *} \frac{\Delta}{\epsilon}\left[\Gamma_{1} \tilde{\rho} f_{1} e^{-i \phi_{1}}+\Gamma_{3} \tilde{\rho} f_{3} e^{-i \phi_{3}}\right]
\end{aligned}
$$




$$
\begin{aligned}
& -i G_{11}^{r *} G_{12}^{r} \frac{\Delta}{\epsilon}\left[\Gamma_{1} \tilde{\rho} f_{1} e^{i \phi_{1}}+\Gamma_{3} \tilde{\rho} f_{3} e^{i \phi_{3}}\right] \\
& +i\left|G_{12}^{r}\right|^{2}\left[\Gamma_{1} \tilde{\rho} f_{1}+\Gamma_{3} \tilde{\rho} f_{3}+\Gamma_{2} \widetilde{f}_{2}+\Gamma_{4} \widetilde{f}_{4}\right], \\
G_{12}^{<}= & i G_{11}^{r} G_{21}^{r *}\left[\Gamma_{1} \tilde{\rho} f_{1}+\Gamma_{3} \tilde{\rho} f_{3}+\Gamma_{2} f_{2}+\Gamma_{4} f_{4}\right] \\
& -i G_{11}^{r} G_{22}^{r *} \frac{\Delta}{\epsilon}\left[\Gamma_{1} \tilde{\rho} f_{1} e^{-i \phi_{1}}+\Gamma_{3} \tilde{\rho} f_{3} e^{-i \phi_{3}}\right] \\
& -i G_{12}^{r} G_{21}^{r *} \frac{\Delta}{\epsilon}\left[\Gamma_{1} \tilde{\rho} f_{1} e^{i \phi_{1}}+\Gamma_{3} \tilde{\rho} f_{3} e^{i \phi_{3}}\right] \\
& +i G_{12}^{r} G_{22}^{r *}\left[\Gamma_{1} \tilde{\rho} f_{1}+\Gamma_{3} \tilde{\rho} f_{3}+\Gamma_{2} \tilde{f}_{2}+\Gamma_{4} \tilde{f}_{4}\right] .
\end{aligned}
$$

Finally, substituting the Green functions $\mathbf{G}^{r}(\epsilon)$, Eq. (21), and $\mathbf{G}^{<}(\boldsymbol{\epsilon})$, Eqs. (28) and (29), into Eqs. (14) and (15), some manipulations (shown explicitly in the Appendix) lead to the current formula of $I^{S 1}$ and $I^{N 2}:{ }^{24}$

$$
I^{S 1}=I_{c}^{S 1}+I_{A}^{S 1}+I_{S}^{S 1}+I_{1}^{S 1}+I_{2}^{S 1}+I_{3}^{S 1}
$$

where

$$
\begin{gathered}
I_{c}^{S 1}=2 e \int \frac{d \epsilon}{2 \pi} \Gamma_{1} \tilde{\rho}\left|G_{11}^{r}\right|^{2}\left[\Gamma_{2}\left(f_{1}-f_{2}\right)+\Gamma_{4}\left(f_{1}-f_{4}\right)\right], \\
I_{A}^{S 1}=-2 e \int \frac{d \epsilon}{2 \pi} \Gamma_{1}\left|G_{12}^{r}\right|^{2} \\
\times \frac{\tilde{\rho} \Gamma_{3}\left(\Gamma_{1}-\Gamma_{3}\right)(1-\cos \phi)+\left(\Gamma_{2}+\Gamma_{4}\right)\left(\Gamma_{1}+\Gamma_{3} \cos \phi\right)}{\Gamma_{1}^{2}+\Gamma_{3}^{2}+2 \Gamma_{1} \Gamma_{3} \cos \phi} \\
\times\left[\Gamma_{2}\left(f_{2}-\tilde{f}_{2}\right)+\Gamma_{4}\left(f_{4}-\tilde{f}_{4}\right)\right], \\
I_{S}^{S 1}=-e \int \frac{d \epsilon}{2 \pi} \Gamma_{1} \Gamma_{3}|A|^{2} \frac{\Delta^{2}}{\Delta^{2}-\epsilon^{2}} \sin \phi \operatorname{Im} \\
\quad \times\left[\Sigma_{11}^{<}\left(g_{22}^{r-1}-\Sigma_{22}^{r}\right)+\Sigma_{22}^{<}\left(g_{11}^{r-1}-\Sigma_{11}^{r}\right)\right], \\
I_{1}^{S 1}=2 e \int \frac{d \epsilon}{2 \pi} \Gamma_{1} \tilde{\rho}\left|G_{12}^{r}\right|^{2}\left[\Gamma_{2}\left(f_{1}-f_{2}\right)+\Gamma_{4}\left(f_{1}-f_{4}\right)\right], \\
I_{2}^{S 1}=-2 e \int \frac{d \epsilon}{2 \pi} \Gamma_{1} \tilde{\rho}_{\frac{2 \Delta}{\epsilon}}^{2} \operatorname{Re}\left[G_{11}^{r} G_{12}^{r *} e^{\left.-i \phi_{1}\right]}\right. \\
\quad \times\left[\Gamma_{2}\left(f_{1}-f_{2}\right)+\Gamma_{4}\left(f_{1}-f_{4}\right)\right], \\
I_{3}^{S 1}=2 e\left(\int_{+\Delta}^{+\infty}+\int_{-\infty}^{--\Delta}\right) \frac{d \epsilon}{2 \pi}|A|^{2} \frac{\epsilon \Delta^{2}}{\epsilon^{2}-\Delta^{2}} \Gamma_{1} \Gamma_{3} \sin \phi \\
\left.\times\left[f_{2}-\tilde{f}_{2}\right)+\Gamma_{4}\left(f_{4}-\tilde{f}_{4}\right)\right],
\end{gathered}
$$

in which $\phi \equiv \phi_{1}-\phi_{3}$ is the phase difference of the two superconducting electrodes, and

$$
I^{N 2}=I_{c}^{N 2}+I_{A}^{N 2}+I_{1}^{N 2}+I_{2}^{N 2},
$$

where

$$
\begin{gathered}
I_{c}^{N 2}=2 e \int \frac{d \epsilon}{2 \pi} \Gamma_{2}\left|G_{11}^{r}\right|^{2}\left[\Gamma_{1} \tilde{\rho}\left(f_{2}-f_{1}\right)\right. \\
\left.+\Gamma_{3} \tilde{\rho}\left(f_{2}-f_{3}\right)+\Gamma_{4}\left(f_{2}-f_{4}\right)\right], \\
I_{A}^{N 2}=2 e \int \frac{d \epsilon}{2 \pi} \Gamma_{2}\left|G_{12}^{r}\right|^{2}\left[\Gamma_{2}\left(f_{2}-\widetilde{f}_{2}\right)+\Gamma_{4}\left(f_{2}-\widetilde{f}_{4}\right)\right], \\
I_{1}^{N 2}=2 e \int \frac{d \epsilon}{2 \pi} \Gamma_{2}\left|G_{12}^{r}\right|^{2}\left[\Gamma_{1} \tilde{\rho}\left(f_{2}-f_{1}\right)+\Gamma_{3} \tilde{\rho}\left(f_{2}-f_{3}\right)\right], \\
I_{2}^{N 2}=-2 e \int \frac{d \epsilon}{2 \pi} \Gamma_{2} \tilde{\rho} \frac{2 \Delta}{\epsilon} \operatorname{Re}\left\{G_{11}^{r} G_{12}^{r *} e^{-i \phi_{1}} \Gamma_{1}\left(f_{2}-f_{1}\right)\right. \\
\left.+G_{11}^{r} G_{12}^{r *} e^{-i \phi_{3}} \Gamma_{3}\left(f_{2}-f_{3}\right)\right\} .
\end{gathered}
$$

The current formulas, Eqs. (30)-(33), are the central results of this work. As a quick check, one can assume $\Gamma_{3}=\Gamma_{4}$ $=0$, which means that the system decouples from the leads $S 3$ and N4, and reduces to the system with a quantum dot connected to a normal and a superconducting lead, the same system as in Ref. 14, then the current formulas $I^{S 1}$ and $I^{N 2}$, Eqs. (30)-(33), reduces exactly to the Eqs. (28) and (29) in Ref. 14.

It is important to notice that the electron occupation number in the quantum dot at the energy $\epsilon$ is given by $n_{e}(\epsilon)$ $=2 \operatorname{Im} G_{11}^{<}(\epsilon),{ }^{25,26}$ and the hole occupation number is $n_{h}(\epsilon)=2 \operatorname{Im} G_{22}^{<}(\epsilon)$. The spectrum function $\rho_{d o t}(\epsilon)$ (i.e., the density of states of quasiparticles in the quantum dot) can be expressed by

$$
\begin{aligned}
\rho_{\mathrm{dot}}(\epsilon)= & n_{e}(\epsilon)+n_{h}(-\epsilon)=2\left[\left|G_{11}^{r}\right|^{2}\right. \\
& \left.+\left|G_{12}^{r}\right|^{2}\right]\left(\Gamma_{1} \tilde{\rho}+\Gamma_{3} \tilde{\rho}+\Gamma_{2}+\Gamma_{4}\right) \\
& -4 \operatorname{Re}\left[G_{11}^{r} G_{12}^{r *} \frac{\Delta}{\epsilon} \tilde{\rho}\left(\Gamma_{1} e^{-i \phi_{1}}+\Gamma_{3} e^{-i \phi_{3}}\right)\right],
\end{aligned}
$$

which is independent of the distribution function of the terminals. Then the normalized distribution function of electrons in the dot is given by $n(\epsilon)=n_{e}(\epsilon) / \rho_{\mathrm{dot}}(\epsilon)$; similarly the distribution function of holes is $\tilde{n}(\epsilon)=n_{h}(\epsilon) / \rho_{\mathrm{dot}}(-\epsilon)$ $=1-n(-\epsilon)$.

Notice that in Eqs. (30)-(33), the currents originating from different types of electron tunneling processes have been separated clearly. For example, let us first look at $I^{N 2}$, which contains four parts: ${ }^{12,14} I_{c}^{N 2}$ comes from the conventional electron tunneling process from $N 2$ to $N 4, S 1$ and $S 3 . I_{A}^{N 2}$ originates from the Andreev reflection, in which an electron incoming from $N 2$ is reflected as a hole going back into $N 2$ or $N 4$, meanwhile an extra Cooper pair is created in the superconducting lead, either in $S 1$ or in $S 3 . I_{1}^{N 2}$ also comes from the Andreev reflection, but the reflected hole exits to the superconducting leads $S 1$ and $S 3$ [notice that due to $V_{1}=V_{3}=0$, so $f_{1}(\epsilon)=\widetilde{f}_{1}(\epsilon)$ and $\left.f_{3}(\epsilon)=\widetilde{f}_{3}(\epsilon)\right] . \quad I_{2}^{N 2}$ originates from the tunneling processes in which an electron incident from $N 2$ tunnels either into $S 1$ or $S 3$, picks up a quasiparticle in the superconductor and creates a Cooper pair. ${ }^{21}$ Second, $I^{S 1}$ contains six parts, in which 
$I_{c}^{S 1}, I_{A}^{S 1}, I_{1}^{S 1}$, and $I_{2}^{S 1}$ originate from the similar processes as the corresponding parts in $I^{N 2}$. In addition, $I_{S}^{S 1}$ is the supercurrent between two superconducting terminals; and $I_{3}^{S 1}$ is from the Andreev reflection, in which a quasielectron (or a quasihole) incoming from $S 1$ will be reflected as a hole (or an electron) going back to $\mathrm{N2}$ or $\mathrm{N4}$. It is worth emphasizing that not only the summation of four terminal currents is zero (i.e., $I^{S 1}+I^{N 2}+I^{S 3}+I^{N 4}=0$, or the total current conservation ${ }^{27}$ ), the summation over terminal index for each type of current is zero as well.

If one assumes that all terminal voltages are zero, then except for $I_{S}^{S 1}$, all types of current (including $I_{c}^{S 1}, I_{A}^{S 1}, I_{1}^{S 1}, I_{2}^{S 1}, I_{3}^{S 1}, I_{c}^{N 1}, I_{A}^{N 1}, I_{1}^{N 1}$, and $\left.I_{2}^{N 1}\right)$ vanish. The supercurrent $I_{S}^{S 1}$ is given by

$$
I_{S}^{S 1}=-2 e \int \frac{d \epsilon}{2 \pi} f_{1}(\epsilon) \Gamma_{1} \Gamma_{3} \sin \phi \frac{\Delta^{2}}{\Delta^{2}-\epsilon^{2}} \operatorname{Im} A^{*} .
$$

In the following three sections we shall apply our current formulas, Eqs. (30)-(33), to investigate the control of the supercurrent and related properties for the four-terminal SNS junction (Sec. III), and the three-terminal SNS junction (Secs. VI and V). In these numerical studies, we take zero temperature $(\mathcal{T}=0)$ and fix the intradot level $\epsilon_{0}=0$. In fact, if $\mathcal{T} \neq 0$ but still with $k_{B} \mathcal{T} \ll \Delta$, the current will not be affected qualitatively: only the supercurrent slightly decreases, while other types of currents have almost no change. The condition of $k_{B} \mathcal{T} \ll \Delta$ is easily realized experimentally, because in typical mesoscopic experiments $\mathcal{T} \leqslant 1 \mathrm{~K}^{1}{ }^{1}$ and the transition temperature of the niobium, which is often used in the experiment, is $9.3 \mathrm{~K}$. Even if $\epsilon_{0}$ slightly deviates zero (but within $\Sigma_{n} \Gamma_{n}$ ), the current still has no qualitative change. However when $\epsilon_{0}$ deviates zero more than several $\Sigma_{n} \Gamma_{n}$, then all types of currents (except for $I_{c}^{N 2}$ ) will strikingly reduce. Therefore, in order to observe these properties experimentally, one can either choose a larger value of $\Sigma_{n} \Gamma_{n}$, or to keep the intradot level $\epsilon_{0}$ at zero by applying a gate voltage.

\section{THE FOUR-TERMINAL CASE}

For the four-terminal SNS junction, we assume that $\Gamma_{1}$ $=\Gamma_{3}$ and $\Gamma_{2}=\Gamma_{4}$ (the symmetric coupling strengths), and $V_{2}=-V_{4}=V / 2$ (the bias voltage $V=2 V_{2}$ ), same as in Refs. 1 and 9. Then the supercurrent in the superconducting terminals dominates. The remaining parts of $I^{S 1}$ (including $I_{c}^{S 1}, I_{A}^{S 1}, I_{1}^{S 1}$, and $I_{3}^{S 1}$ ) vanish, due to the fact that the current from electrons incoming from $N 2$ cancels completely with the current from the holes incoming from $N 4$; however, $I_{2}^{S 1}$ may be either zero if $\left|V_{2}\right|<\Delta$, or nonzero but much smaller than the supercurrent $I_{S}^{S 1}$ if $\left|V_{2}\right|>\Delta$. Meanwhile, all types of current in the normal terminals can be nonzero. In the following, we investigate the supercurrent and normal current, respectively.

Figure 2 presents $I_{S}^{S 1}$ vs $V_{2}$ at different coupling strengths $\Gamma_{2}$ and phase differences $\phi$. One can see (1) at $V_{2}=0$, the supercurrent $I_{S}^{S 1}$ reaches its maximum. With the increase of $V_{2}$, the supercurrent $I_{S}^{S 1}$ reduces drastically. (2) While $V_{2}$ exceeds a certain critical value $V_{2 c}$, (e.g., for $\Gamma_{2}=\Gamma_{4}$ $\left.=0.2 \Delta, V_{2 c} \sim 0.36 \Delta\right), I_{S}^{S 1}$ becomes negative, namely, the

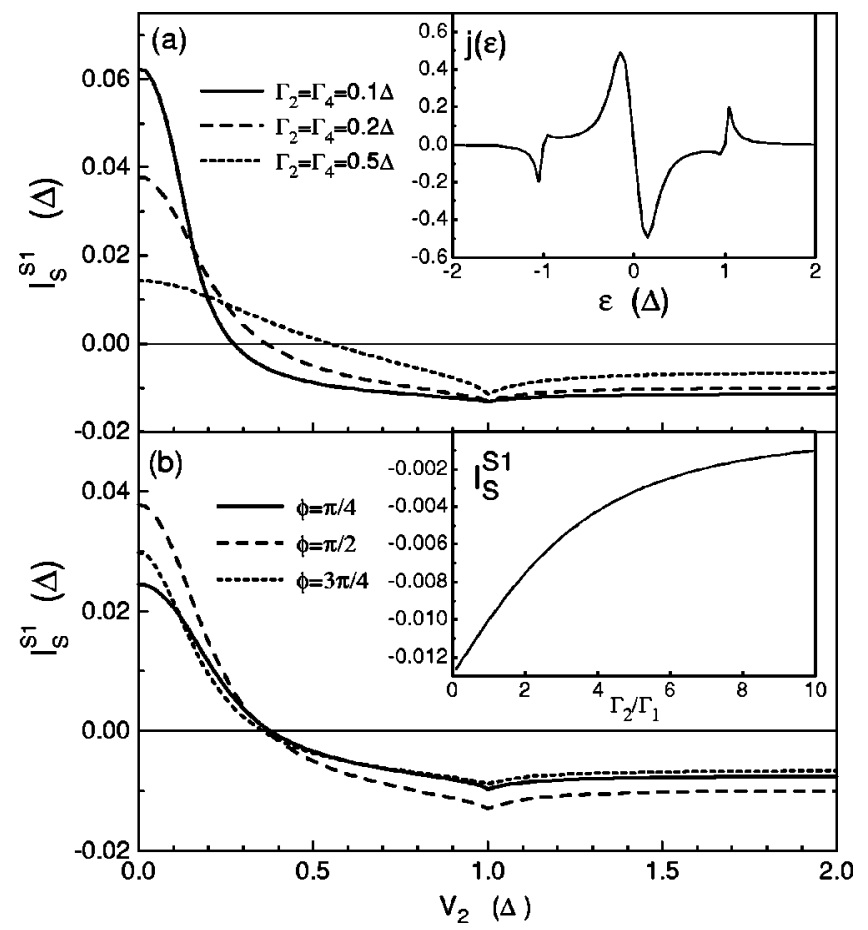

FIG. 2. (a) The supercurrent $I_{S}^{S 1}$ vs $V_{2}$ at different $\Gamma_{2}=\Gamma_{4}$, for a fixed phase difference $\phi=\pi / 2$, and other parameters are $\Gamma_{1}=\Gamma_{3}$ $=0.2 \Delta, \epsilon_{0}=0 \quad\left(\epsilon_{0}=0\right.$ for all figures $)$. The inset shows $j(\epsilon)$ vs $\epsilon$ for the parameters $\Gamma_{1}=\Gamma_{2}=\Gamma_{3}=\Gamma_{4}=0.2 \Delta$ and $\phi=\pi / 2$. (b) The supercurrent $I_{S}^{S 1}$ vs $V_{2}$ at different $\phi$, for $\Gamma_{1}=\Gamma_{2}=\Gamma_{3}=\Gamma_{4}$ $=0.2 \Delta$. The inset shows $I_{S}^{S 1}$ vs $\Gamma_{2} / \Gamma_{1}$ at $V_{2}=2 \Delta$ and $\phi=\pi / 2$.

SNS junction transits to a $\pi$ junction. The critical value $V_{2 c}$ is dependent on $\Gamma_{2}$ [Fig. 2(a)], but almost independent of $\phi$ [Fig. 2(b)]. Figure 3 shows $I_{S}^{S 1}$ vs $\phi$ at different $V_{2}$. When $V_{2}<V_{2 c}$, the SNS junction exhibits the conventional current-phase relation $I_{S}^{S 1} \sim\left(I_{S}^{S 1}\right)_{c} \sin \phi$. While $V_{2}>V_{2 c}$, a transition to the $\pi$ junction occurs with the current-phase relation $I_{S}^{S 1} \sim\left(I_{S}^{S 1}\right)_{c} \sin (\phi+\pi)$. At $V_{2}=V_{2 c}, I_{S}^{S 1}$ vanishes for any phase difference $\phi$. (3) When $V_{2}=\Delta$, the negative supercurrent reaches its largest magnitude; then with the further increase of $V_{2}$, the absolute value of the negative supercurrent decreases slowly, but does not vanish. All these results are consistent with the experiment by Baselmans et al., ${ }^{1}$

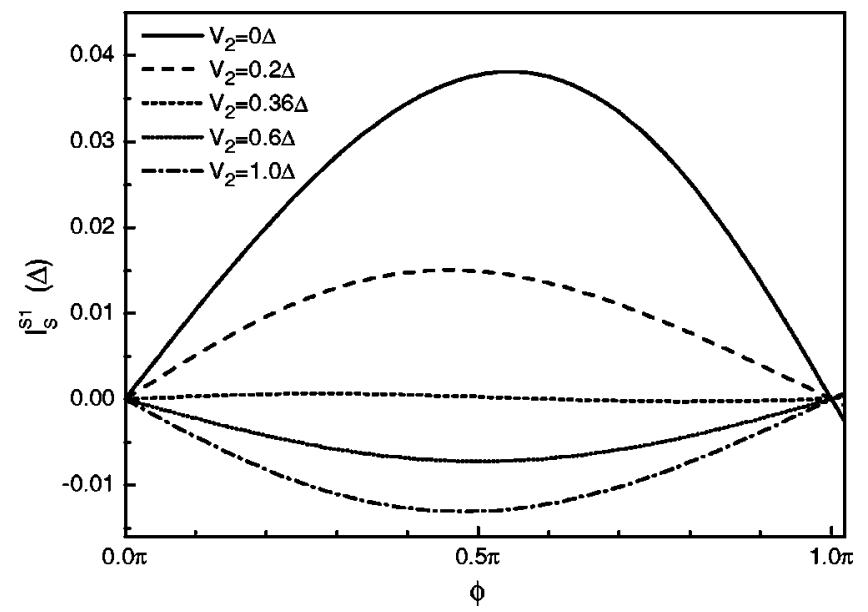

FIG. 3. $I_{S}^{S 1}$ vs $\phi$ at different $V_{2}$, for $\Gamma_{1}=\Gamma_{2}=\Gamma_{3}=\Gamma_{4}=0.2 \Delta$. 


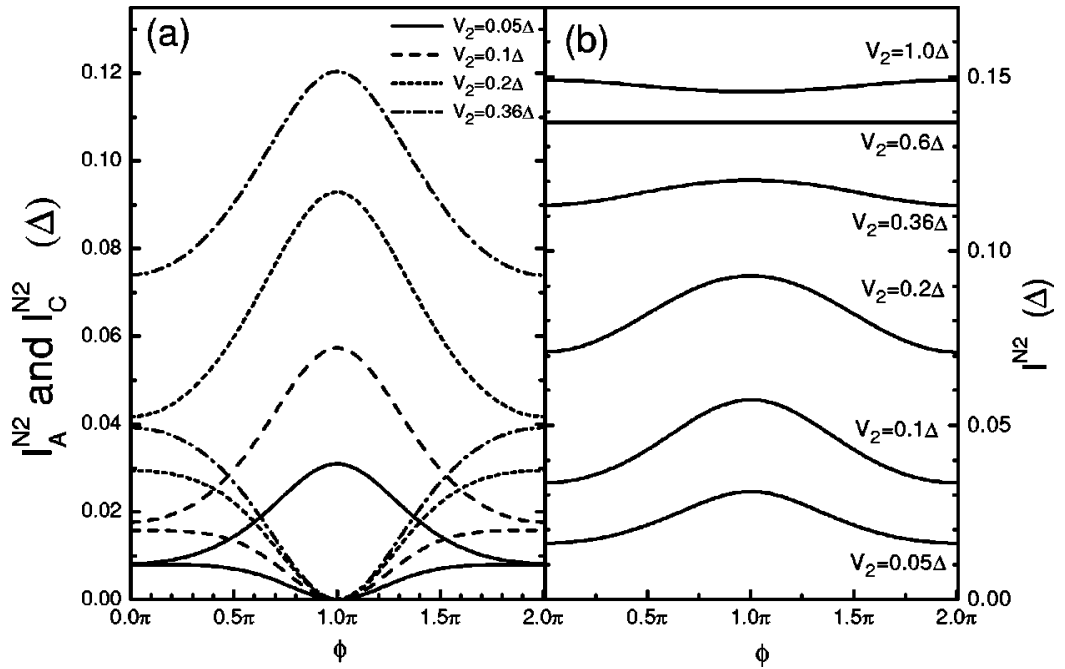

FIG. 4. (a) $I_{A}^{N 2}$ and $I_{c}^{N 2}$ vs $\phi$ at different $V_{2}$. The curves with zero value at $\phi=\pi$ represent $I_{A}^{N 2}$. (b) The total current $I^{N 2}$ vs $\phi$ at different $V_{2}$. Other parameters are: $\Gamma_{1}=\Gamma_{2}=\Gamma_{3}=\Gamma_{4}$ $=0.2 \Delta$. indicating that the SNS junction, in which the $N$ region is a quantum dot, still has similar behavior in controlling the supercurrent as in the diffusive SNS junction.

In order to explain the properties of the supercurrent of the SNS junction, we rewrite the supercurrent in the form as

$$
I_{S}^{S 1}=e \int \frac{d \epsilon}{2 \pi} j(\epsilon)\left[\frac{\Gamma_{1}}{\Gamma_{2}} \tilde{\rho}\left(f_{1}+f_{3}\right)+f_{2}+\widetilde{f}_{2}\right],
$$

where

$$
j(\epsilon)=-\Gamma_{1}^{2} \Gamma_{2} \sin \phi \frac{|A|^{2} \Delta^{2}}{\Delta^{2}-\epsilon^{2}} \operatorname{Re}\left[g_{11}^{r-1}-\Sigma_{11}^{r}+g_{22}^{r-1}-\Sigma_{22}^{r}\right],
$$

in which we have used $f_{2}(\epsilon)=\widetilde{f}_{4}(\epsilon)$ (due to $V_{2}=$ $\left.-V_{4}\right), \Gamma_{1}=\Gamma_{3}$, and $\Gamma_{2}=\Gamma_{4} \cdot j(\epsilon)$ is the energy-dependent supercurrent-carrying density of states, shown in the inset of Fig. 2(a) ${ }^{5,8,28}$ In the gap region, the Andreev quasibound states $\epsilon_{A}^{+}$and $\epsilon_{A}^{-}$[see Eq. (38) of Sec. IV] are responsible for $j(\epsilon)$ : for $-\Delta<\epsilon<0, j(\epsilon)$ is positive, originated from the Andreev quasibound state $\epsilon_{A}^{+}$, and reaches maximum at $\epsilon$ $=\epsilon_{A}^{+}$; while for $0<\epsilon<\Delta, j(\epsilon)$ is negative, originated from the Andreev quasibound state $\epsilon_{A}^{-}$, and reaches maximum at $\epsilon=\epsilon_{A}^{-}$. Since we have assumed only one single level in the dot, only a pair of Andreev quasibound states $\epsilon_{A}^{ \pm}$exists. In contrast, outside the gap region $(|\epsilon|>\Delta)$, the continuous spectrum is responsible for $j(\epsilon)$, with negative value for $\epsilon$ $<-\Delta$, and positive for $\epsilon>\Delta$. Since we assumed that $V_{2}=$ $-V_{4}$, we can easily obtain the distribution function of electrons in the dot as $n(\epsilon)=\left[\left(\Gamma_{1} / \Gamma_{2}\right) \tilde{\rho}\left(f_{1}+f_{3}\right)+f_{2}\right.$ $\left.+f_{4}\right] /\left[2\left(\Gamma_{1} / \Gamma_{2}\right) \tilde{\rho}+2\right]$, which has a nonequilibrium twostep form for $|\epsilon|<\Delta$. The behavior of $I_{S}^{S 1}$ is determined by the combination of the supercurrent-carrying density of states $j(\epsilon)$ and the distribution function of electrons in the dot. For example, at $V_{2}=0$, electrons in the dot occupy the states with $\epsilon<0$, leading to the largest value of $I_{S}^{S 1}$. With the increase of $V_{2}$, the distribution of electrons in the dot becomes a nonequilibrium two-step form, ${ }^{11}$ leading to a significant cancellation of the supercurrent carried by Andreev quasibound levels $\epsilon_{A}^{+}$and $\epsilon_{A}^{-}$, and correspondingly a notably decrease of $I_{S}^{S 1}$, which may even reach negative value if
$V_{2} \geqslant V_{2 c}$. While for $V_{2}=\Delta$, the contributions from $\epsilon_{A}^{+}$and $\epsilon_{A}^{-}$cancel each other completely, so the supercurrent originates only from the continuous spectrum of $\epsilon<-\Delta$, which is negative. Also notice that $I_{S}^{S 1}$ does not approach zero at large $V_{2}$ (e.g., $V_{2}=2.0$ ), due to the fact that the intradot distribution function at $|\epsilon|>\Delta$ also depends on the distribution function of quasiparticles in superconducting terminals, i.e., $f_{1}$ and $f_{3}$. With the increase of $\Gamma_{2} / \Gamma_{1}, n(\epsilon)$ and $\tilde{n}(\epsilon)$ will mainly be determined by two normal terminals for $|\epsilon|$ $>\Delta$, then $I_{S}^{S 1}$ at large $V_{2}$ will go to zero [see inset of Fig. 2(b)].

Now we study the current $I^{N 2}$ flowing into the dot from the normal terminal $N 2$. If $\left|V_{2}\right|<\Delta$, only $I_{c}^{N 2}$ and $I_{A}^{N 2}$ are nonzero. But for $\left|V_{2}\right|>\Delta$, all types of current are nonzero. Figure 4(a) shows $I_{c}^{N 2}$ and $I_{A}^{N 2}$ vs $\phi$ at different bias $V_{2}$. Although the dependence of the Andreev reflection current with $\phi$ has been investigated extensively in systems with two superconductor reflection mirrors; ${ }^{16,29,30}$ much less attention has been paid for studying the dependence of the conventional current with $\phi$. In our four-terminal system, the current-phase relation of $I_{A}^{N 2}$ can be fitted very well with $I_{A}^{N 2}(\phi)=I_{A}^{N 2}(0)(1+\cos \phi) / 2$, due to the fact that an electron, incoming from terminal $N 2$ with energy $\epsilon$, will be reflected by either the superconductor $S 1$ or $S 3$ [see Fig. 5(a)]. Notice that the phase shift by the Andreev reflection is $\phi_{n}$

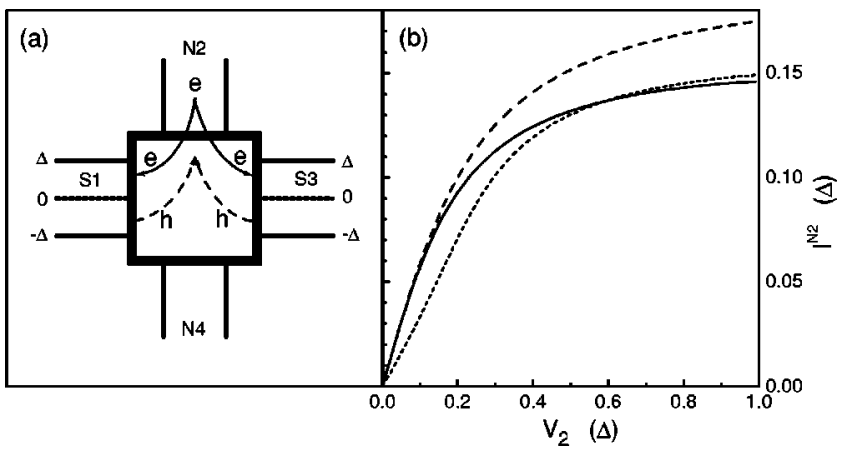

FIG. 5. (a) A schematic diagram for the Andreev reflection current $I_{A}^{N 2}$ in Fig. 4(a). (b) $I^{N 2}$ vs $V_{2}$ for $\phi=0$ (dotted curve) and for $\phi=\pi$ (solid curve). Other parameters are the same as in Fig. 4(b). The dashed curve shows $I^{N 2}$ vs $V_{2}$ without two superconducting terminals for comparison. 
$+\arccos (\epsilon / \Delta) \quad(n=1,3)$, in which the first part depends on the superconducting phase $\phi_{n} \cdot{ }^{14}$ Therefore if $\phi=\phi_{1}-\phi_{3}$ $=0$, the Andreev reflected holes going back from $S 1$ and $S 3$ experience a constructive interference, leading to the largest value of $I_{A}^{N 2}$. On the other hand, if $\phi=\pi$, the reflected holes from $S 1$ and $S 3$ have a destructive interference, resulting the smallest $I_{A}^{N 2}$. For symmetric barriers $\left(\Gamma_{1}=\Gamma_{3}\right)$, the amplitudes of Andreev reflected holes by $S 1$ and $S 3$ are equal, hence $I_{A}^{N 2}(\pi)$ vanishes.

The conventional current $I_{c}^{N 2}$ vs $\phi$ is also shown in Fig. 4(a), in which all curves can approximately be fitted with the current-phase relation $I_{c}^{N 2}(\phi)=I_{c}^{N 2}(0)(1+\alpha \cos \phi) /(1+\alpha)$ with $-1<\alpha<0$, where $I_{c}^{N 2}$ has the smallest value if $\phi=0$; and the largest value if $\phi=\pi$. This behavior is completely opposite to the Andreev reflection current $I_{A}^{N 2}$. The reason is, for $\phi=0, I_{A}^{N 2}$ reaches its largest value, indicating that the majority of electrons incoming from $N 2$ participates in the Andreev reflection, or equivalently, the minority of electrons participates in the conventional tunneling, leading to the smallest value of $I_{c}^{N 2}$. On the contrary, if $\phi=\pi$, the Andreev reflection is suppressed, almost all electrons incoming from terminal $N 2$ participate in the conventional tunneling, resulting in the largest value of $I_{c}^{N 2}$.

The dependence of $I^{N 2}$ on $\phi$ at difference $V_{2}$ is given in Fig. 4(b). For $V_{2} \leqslant \Delta, I^{N 2}=I_{A}^{N 2}+I_{c}^{N 2}$, where $I^{N 2}$ can also be approximately fitted with the form $I^{N 2}(\phi)=I^{N 2}(0)(1$ $+\alpha \cos \phi) /(1+\alpha)$. In this case, the factor $\alpha$ may either be positive or negative, i.e., $I^{N 2}(\pi)$ may either be larger than $I^{N 2}(0)$, if $V_{2}>0.6 \Delta$; or smaller than $I^{N 2}(0)$, if $V_{2}<0.6 \Delta$, due to the fact that the current-phase relations of $I_{c}^{N 2}$ and $I_{A}^{N 2}$ are just opposite. In fact, the change of $I^{N 2}$ with $\phi$ is not noticeable. The dependence of $I^{N 2}(0)$ and $I^{N 2}(\pi)$ on $V_{2}$ is shown in Fig. 5(b). The difference between the two curves is small. For comparison, the current $I^{N 2}$ for a system decoupled from two superconducting terminals (by setting $\Gamma_{1}$ $\left.=\Gamma_{3}=0\right)$ is also shown in Fig. 5(b).

\section{THE THREE-TERMINAL CASE}

There have been several theoretical studies on threeterminal SNS junctions. Wees et al. ${ }^{2}$ studied the dependence of the supercurrent on the temperature and the coupling strength between the normal terminal and the SNS junction. Chang and Bagwell investigated the control of the Josephson current by the normal probe bias voltage $V_{2}$, and also proposed a method to measure the continuous spectrum which contributes to the Josephson current. ${ }^{4}$ Ilhan, Demir, and Bagwell studied the Andreev-level spectroscopy and Josephsoncurrent switching in a three-terminal SIS junction. ${ }^{31}$ The three-terminal SNS junction investigated in this section is deduced from our four-terminal SNS junction by decoupling the normal terminal $N 4$ from the system (simply by setting $\Gamma_{4}=0$ ), so we can directly use our current formulas obtained in Sec. II. However, the following investigations are different from the work in Refs. 2,4,31 in the following aspects: (1) The $N$ region in this work is a quantum dot, connected to superconducting terminals through barriers; while in previous works, the $N$ region is either a ballistic $2 \mathrm{DEG}$ or a diffusive normal metal, and without barriers separating the central $N$ region and the superconducting terminals. (2) Instead

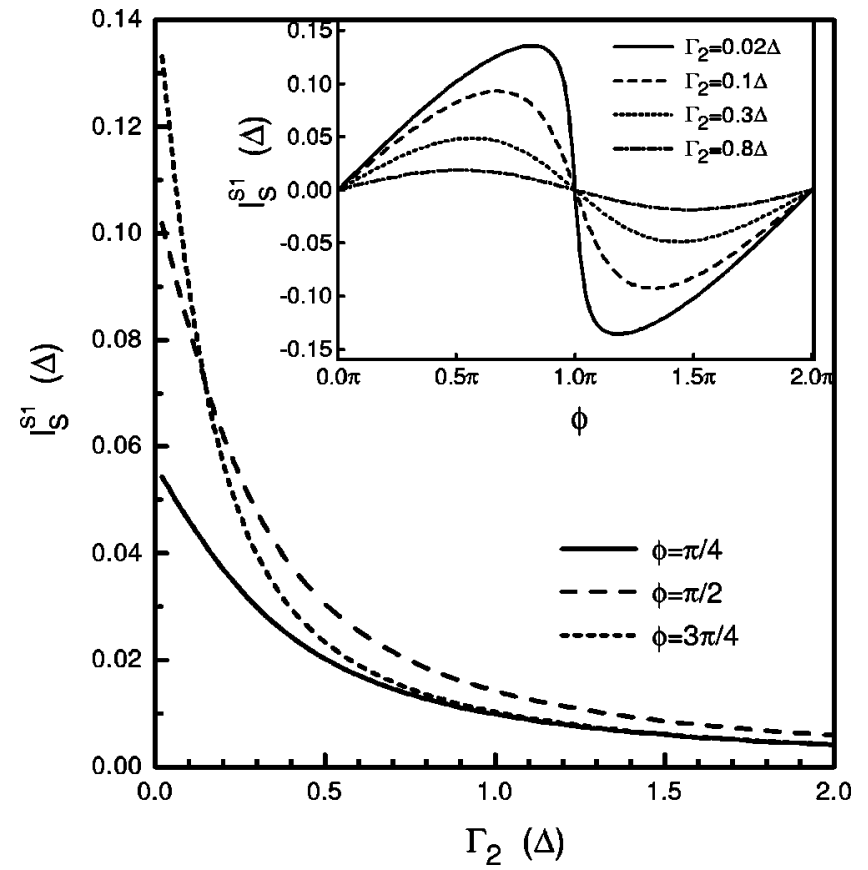

FIG. 6. $I_{S}^{S 1}$ vs $\Gamma_{2}$ for the three-terminal SNS junction. Other parameters are $\Gamma_{1}=\Gamma_{3}=0.2 \Delta, V_{2}=0$. The inset shows $I_{S}^{S 1}$ vs $\phi$ at different $\Gamma_{2}$.

of using Bogoliubov-de Gennes equation and the scattering matrix theory, the nonequilibrium Green's function method has been used here. (3) In addition to the supercurrent, we also investigate in detail the Andreev reflection current $I_{A}$ in each terminal. In the following, we first study the case of $V_{2}=0$, then the case of $V_{2} \neq 0$.

$$
\text { A. } V_{2}=0
$$

In the case of $V_{2}=0$ for the three-terminal SNS junction, there is obviously no net current flowing into the dot from the normal terminal; only the supercurrent $I_{S}^{S 1}$ exists. However, the existence of the normal terminal can still affect the supercurrent. Based on Eq. (35), the dependence of the supercurrent $I_{S}^{S 1}$ on the coupling strength $\Gamma_{2}$ at different $\phi$ is calculated and shown in Fig. 6. It is clearly seen that the supercurrent $I_{S}^{S 1}$ is suppressed with the increase of the coupling strength $\Gamma_{2}$, as pointed out by Chang and Bagwell. ${ }^{4} I_{S}^{S 1}$ vs $\phi$ at different $\Gamma_{2}$ is given in the inset of Fig. 6, showing the reduction of the critical supercurrent with the increase of $\Gamma_{2}$. It also shows a sinelike current-phase relation of $I_{S}^{S 1}$ for large $\Gamma_{2}$; but a significant deviation from the sinelike behavior for small $\Gamma_{2}{ }^{8}$ The reason for the suppression of the supercurrent is: for large $\Gamma_{2}$, it is much easier for electrons to tunnel from the quantum dot to the normal lead $N 2$ and be randomized in the normal reservoir. Therefore electrons, traversing through the dot from one superconducting terminal to another, have a much higher probability to lose the phase memory, leading to a suppression of the supercurrent. As a result, one can control the supercurrent by changing the coupling strength between the dot and the normal terminal N2. Since there is no net normal current from the dot to the normal terminal $N 2$, this way of controling the supercurrent is different from the schemes suggested previously, in which the main ideas are either to control the electron density in the 


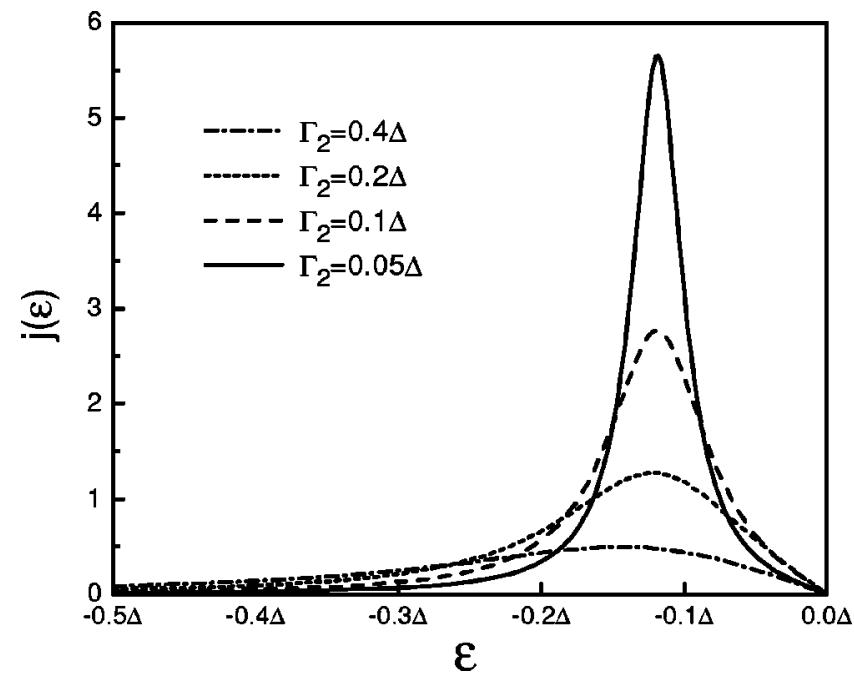

FIG. 7. The supercurrent density $j(\epsilon)$ vs $\epsilon$ at different $\Gamma_{2}$, with $\Gamma_{1}=\Gamma_{3}=0.2 \Delta$ and $\phi=\pi / 2$.

central $N$ region by tuning the gate voltage; ${ }^{16,32}$ or to change the distribution of quasiparticles in the $N$ region by a normal current flowing through it. ${ }^{1,9,10}$ The scheme suggested in this work for controlling the supercurrent may have the following advantage: since no current flows from the normal terminal, it should have no heat dissipation, which is important to maintain the operation of the device at low temperature.

The supercurrent-carrying density of states $j(\epsilon)$ is presented in Fig. 7, in which a peak emerges at $\epsilon \sim 0.12 \Delta$, originated from the Andreev quasibound state $\epsilon_{A}^{+}$. With the increase of $\Gamma_{2}$, the peak becomes wider but lower, due to the spreading of the Andreev quasibound state. In addition, from the behavior of $j(\epsilon)$, the suppression of the supercurrent $I_{S}^{S 1}$ with the increase of $\Gamma_{2}$ can easily be understood.

\section{B. $V_{2} \neq 0$}

For $\left|V_{2}\right|<\Delta$, both $I_{S}^{S 1}$ and $I_{A}^{S 1}$ exist in the superconducting terminals; but only $I_{A}^{N 2}$ exists in the normal terminal $N 2$. While for $\left|V_{2}\right|>\Delta$, all types of currents emerge. The dependences of all types of currents in terminal $S 1$ on $V_{2}$ are presented in Fig. 8. One can clearly see that (1) the supercurrent $I_{S}^{S 1}$ is suppressed and the SNS junction changes to a $\pi$ junction at a certain value of $V_{2}$, similar to the fourterminal SNS junction case discussed above. However, in the four-terminal case, only $I_{S}^{S 1}$ flows into the dot from terminal $S 1$; while in the three-terminal case, all types of currents exist, therefore the supercurrent can not be measured separately. (2) The magnitude of the Andreev reflection current $I_{A}^{S 1}$ first increases quickly, then almost maintains a constant value, as will be discussed later. (3) The conventional current $I_{c}^{S 1}$ is zero for $\left|V_{2}\right| \leqslant \Delta$, and decreases slowly when $V_{2}$ $\geqslant \Delta$. (4) $I_{1}^{S 1}, I_{2}^{S 1}$, and $I_{3}^{S 1}$ are very small. In Fig. 8, we have multiplied $I_{1}^{S 1}$ and $I_{2}^{S 1}$ by a factor of 20 and 50, respectively, for clarity. $I_{3}^{S 1}$ is zero for $\epsilon_{0}=0$, due to the complete cancellation of the quasiparticles incoming from $S 1$ and $S 3$.

Now we study the Andreev reflection current and the Andreev quasibound states $\epsilon_{A}^{ \pm}$which have been mentioned above. ${ }^{33}$ In fact, the Andreev quasi-bound states $\epsilon_{A}^{ \pm}$are related to both the Andreev reflection current and the supercur-

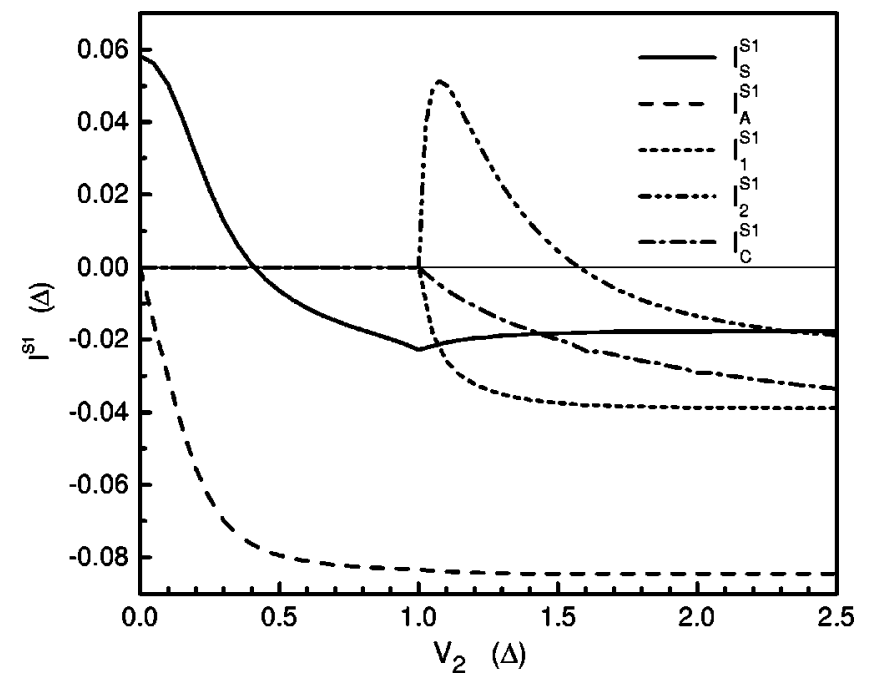

FIG. 8. The current $I_{S}^{S 1}, I_{A}^{S 1}, I_{1}^{S 1}, I_{2}^{S 1}$, and $I_{c}^{S 1}$ vs $V_{2}$, for $\Gamma_{1}$ $=\Gamma_{3}=0.3 \Delta, \Gamma_{2}=0.5 \Delta$, and $\phi=\pi / 2$. For clarity, $I_{1}^{S 1}$ and $I_{2}^{S 1}$ have been multiplied by a factor of 20 and 50 , respectively.

rent. $\epsilon_{A}^{ \pm}$can be obtained by finding the extremum of the spectrum function $\rho_{\mathrm{dot}}(\epsilon)$ in the quantum dot. Notice that if no superconductor exists (i.e, let $\Gamma_{1}=\Gamma_{3}=0$ ), the original bound state of the dot is at obviously $\epsilon_{0}$, as assumed. Now in the presence of the superconductors $S 1$ and $S 3$, the original level $\epsilon_{0}$ will be split into two Andreev quasi-bound states $\epsilon_{A}^{ \pm}$, determined by the superconducting phase different $\phi$ and the coupling strengths $\Gamma_{1}$ and $\Gamma_{3}$. When $\Gamma_{2} \ll \Delta$, the energies $\epsilon_{A}^{ \pm}$are approximately independent of $\Gamma_{2}$, then it can be obtained from the equation $\operatorname{Re}\left[A\left(\epsilon_{A}^{ \pm}\right)\right]^{-1}=0$, as

$$
\left[\epsilon_{A}^{ \pm}-\sum_{n=1,3} \sum_{n ; 11}^{r}\left(\epsilon_{A}^{ \pm}\right)\right]-\epsilon_{0}^{2}-\Sigma_{12}^{r}\left(\epsilon_{A}^{ \pm}\right) \Sigma_{21}^{r}\left(\epsilon_{A}^{ \pm}\right)=0 .
$$

The linewidth (or the coupling strength) $\Gamma_{2}$ only determines the spreading of the Andreev quasibound state. When the energy $\epsilon$ of an incoming electron lines up with the Andreev quasibound states $\epsilon_{A}^{ \pm}$, a resonance will occur, leading to a very large Andreev reflection current. In the following we will concentrate on the case of $V_{2} \leqslant \Delta$, under this condition only the Andreev current $I_{A}^{N 2}$ exists in $N 2$ and can be measured easily (while for $V_{2} \geqslant \Delta$, it is impossible to measure $I_{A}^{N 2}$ in terminal $N 2$, because all types of current emerge). Figure 9(a) shows the Andreev reflection current $I_{A}^{N 2}$ vs $V_{2}$ at different $\Gamma_{2}$. All curves exhibit a steplike pattern, and the position of the steps are located near $V_{2} \sim 0.22 \Delta$, i.e., near the position of the Andreev bound state $\epsilon_{A}^{+}$. If $V_{2} \geqslant \epsilon_{A}^{+}$, the Fermi level of the normal terminal $N 2$ will be higher than $\epsilon_{A}^{+}$, the electron incoming from $N 2$ may have the energy $\epsilon$ $=\epsilon_{A}^{+}$, so a resonance occurs; and a Andreev reflected hole with the energy $\epsilon=\epsilon_{A}^{-}$is created, leading to a large Andreev reflection current. Therefore, when $V_{2}$ varies through $\epsilon_{A}^{+}, I_{A}^{N 2}$ increases sharply; but for other values of $V_{2}$, it almost does not change. It should be mentioned that at different values of $\Gamma_{2}$, the slopes of different curves of $I_{A}^{N 2}$ at $V_{2}=\epsilon_{A}^{+}$are not the same. With larger $\Gamma_{2}$, the rising slope is smaller. This means that the half-width of $\epsilon_{A}^{ \pm}$is definitely 

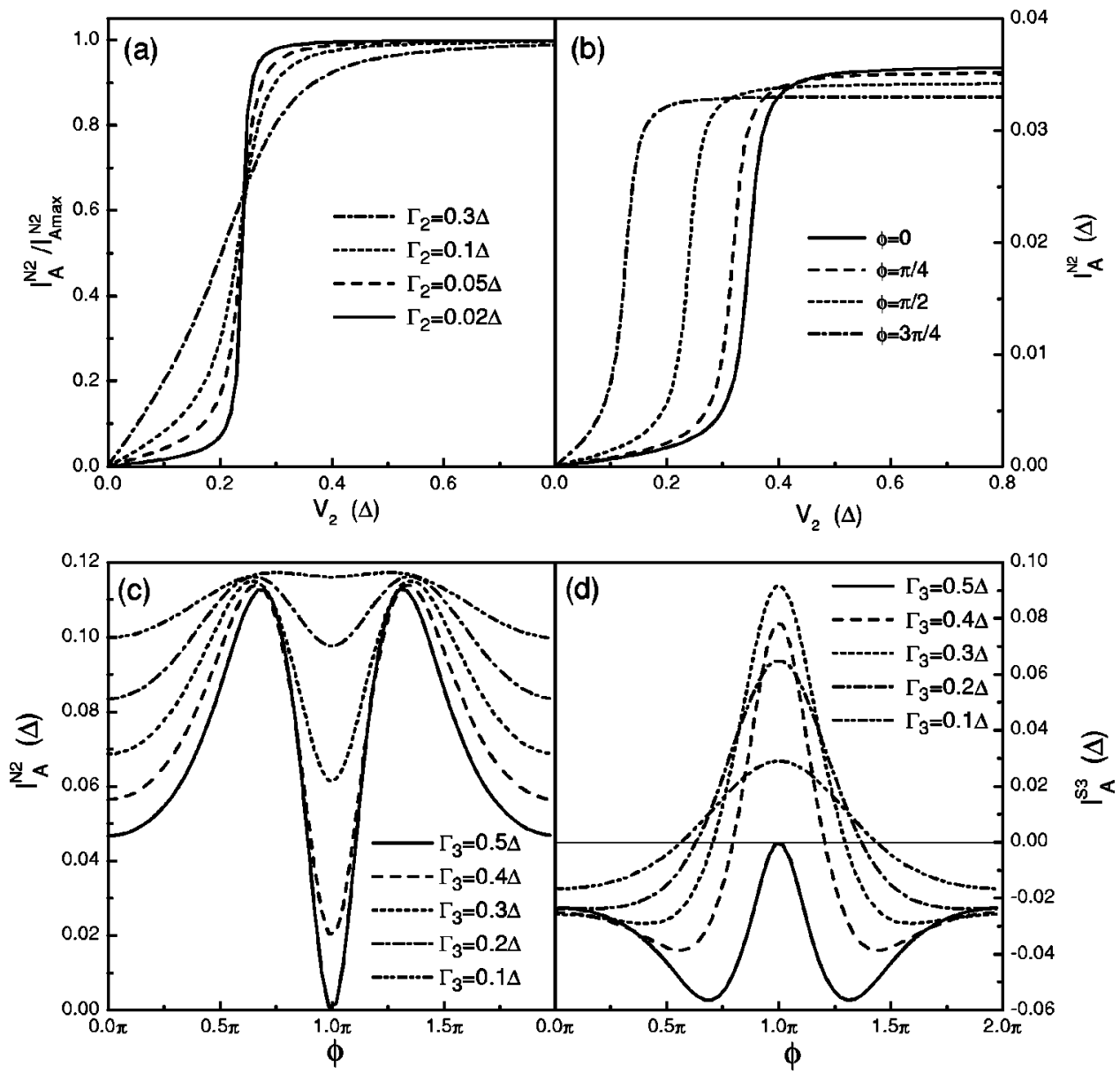

FIG. 9. (a) $I_{A}^{N 2} / I_{A}^{N 2} \max$ vs $V_{2}$ at different $\Gamma_{2}$, where $I_{A \text { max }}^{N 2}=I_{A}^{N 2}\left(V_{2}\right.$ $=\infty)$. Other parameters are $\Gamma_{1}$ $=\Gamma_{3}=0.5 \Delta$ and $\phi=\pi / 2$. (b) $I_{A}^{N 2}$ vs $V_{2}$ at different $\phi$, for $\Gamma_{1}=\Gamma_{3}$ $=0.5 \Delta$ and $\Gamma_{2}=0.05 \Delta$. (c) $I_{A}^{N 2}$ vs $\phi$ at different $\Gamma_{3}$. (d) $I_{A}^{S 3}$ vs $\phi$ at different $\Gamma_{3}$. The parameters in (c) and (d) are $\Gamma_{1}=0.5 \Delta, \Gamma_{2}$ $=0.3 \Delta$, and $V_{2}=0.2 \Delta$. determined by $\Gamma_{2}$. In Fig. 9(b), we show the Andreev reflection current $I_{A}^{N 2}$ vs $V_{2}$ at different $\phi$. One finds that the position of the step, i.e., the value of $\epsilon_{A}^{+}$, is shifted for different $\phi$. For $\phi=0, \epsilon_{A}^{+}$has the largest shift; while for $\phi$ $=\pi, \epsilon_{A}^{+}$has no shift at all. This means that the positions of $\epsilon_{A}^{ \pm}$depend on the phase difference $\phi$, which can also be obtained by solving Eq. (38). It should be pointed out that we have set $\epsilon_{0}=0$ in the above numerical calculation. If $\epsilon_{0}$ $\neq 0$, then $\epsilon_{A}^{+} \neq-\epsilon_{A}^{-}$, and the Andreev reflection will not be on resonance, so the Andreev reflection current will reduce significantly.

The current-phase relation of the Andreev reflection current $I_{A}^{N 2}$ is shown in Fig. 9(c) for $V_{2}=0.2 \Delta$. The curves exhibit quite complicated pattern due to two factors: (1) the coherent effect between the Andreev reflected holes by $S 1$ and $S 3$ (see Sec. III), (2) the energy shift of the Andreev quasibound state $\epsilon_{A}^{ \pm}$depends on $\phi$. First, let us look at curves of $I_{A}^{N 2}$ vs $\phi$ in Fig. 9(c). At $\phi=\pi$, since Andreev reflected holes by $S 1$ and $S 3$ are out of phase, it results to a destructive interference and a very small $I_{A}^{N 2}$. When $\phi$ deviates from $\pi$, the effect of destructive interference becomes weaker and weaker, leading to an increase of $I_{A}^{N 2}$. While $\phi$ exceeds a certain value (about $0.65 \pi$ ), the Andreev quasibound state $\epsilon_{A}^{+}$becomes larger than $V_{2}=0.2 \Delta$, then no electron incoming from $N 2$ can reach the energy $\epsilon_{A}^{+}$, therefore $I_{A}^{N 2}$ becomes smaller again. Figure 9(c) also shows $I_{A}^{N 2}$ vs $\phi$ at different $\Gamma_{3}$. With the decrease of $\Gamma_{3}, I_{A}^{N 2}$ has a weaker dependence on $\phi$. In the limit of $\Gamma_{3} \rightarrow 0$, both $I_{A}^{N 2}$ and $\epsilon_{A}^{+}$ will be independent of $\phi$, because the superconducting terminal $S 3$ is decoupled from the system, and the system simply reduces to a two terminal $S$-QD- $N$ system with a superconducting terminal $S 1$ and a normal terminal $N 2$.

It is interesting to know how the Andreev current $I_{A}^{N 2}$ flowing into the dot from the normal terminal will be distributed between the two superconducting terminals $S 1$ and $S 3$ (Notice: $I_{A}^{S 1}+I_{A}^{S 3}=-I_{A}^{N 2}$ ). We present the Andreev current $I_{A}^{S 3}$ vs $\phi$ at different $\Gamma_{3}$ in Fig. $9(\mathrm{~d}) . I_{A}^{N 2}$ have been shown in Fig. 9(c), and $I_{A}^{S 1}$ can be easily obtained from $I_{A}^{S 1}=-I_{A}^{N 2}$ $-I_{A}^{S 3}$. When $\Gamma_{1}=\Gamma_{3}$, the Andreev current $I_{A}^{S 1}$ is equal to $I_{A}^{S 3}$, which means that in the Andreev reflection process, an electron incoming from $N 2$ will have the same probability reflecting back as a hole either by $S 1$ or by $S 3$, so the same number of the Cooper pairs are created in the two superconducting terminals. While $\Gamma_{1} \neq \Gamma_{3}$ (e.g., $\Gamma_{1}>\Gamma_{3}$ ), the difference between the Andreev currents $I_{A}^{S 1}$ and $I_{A}^{S 3}$ may become significant, they may even have different directions. One can see from Fig. 9 that $I_{A}^{S 3}$ may be positive, i.e., in the Andreev reflection process, no creation but annihilation of Cooper pairs in the superconductor $S 3$. It is of merit to mention that for $\left|V_{2}\right| \leqslant \Delta, I_{A}^{S 3} / I_{A}^{S 1}$ is exactly equal to $\Gamma_{3}\left(\Gamma_{3}\right.$ $\left.+\Gamma_{1} \cos \phi\right) / \Gamma_{1}\left(\Gamma_{1}+\Gamma_{3} \cos \phi\right)$. When $\Gamma_{3} \rightarrow 0, I_{A}^{S 3}$ will also approach to zero, due to the decoupling between the superconducting terminal $S 3$ from the system.

\section{THE LIMIT CASE OF $\Gamma_{2} \rightarrow 0$}

In the limit of $\Gamma_{2} \rightarrow 0$, the three-terminals SNS junction tends to decouple from the normal terminal $N 2$, and reduces 

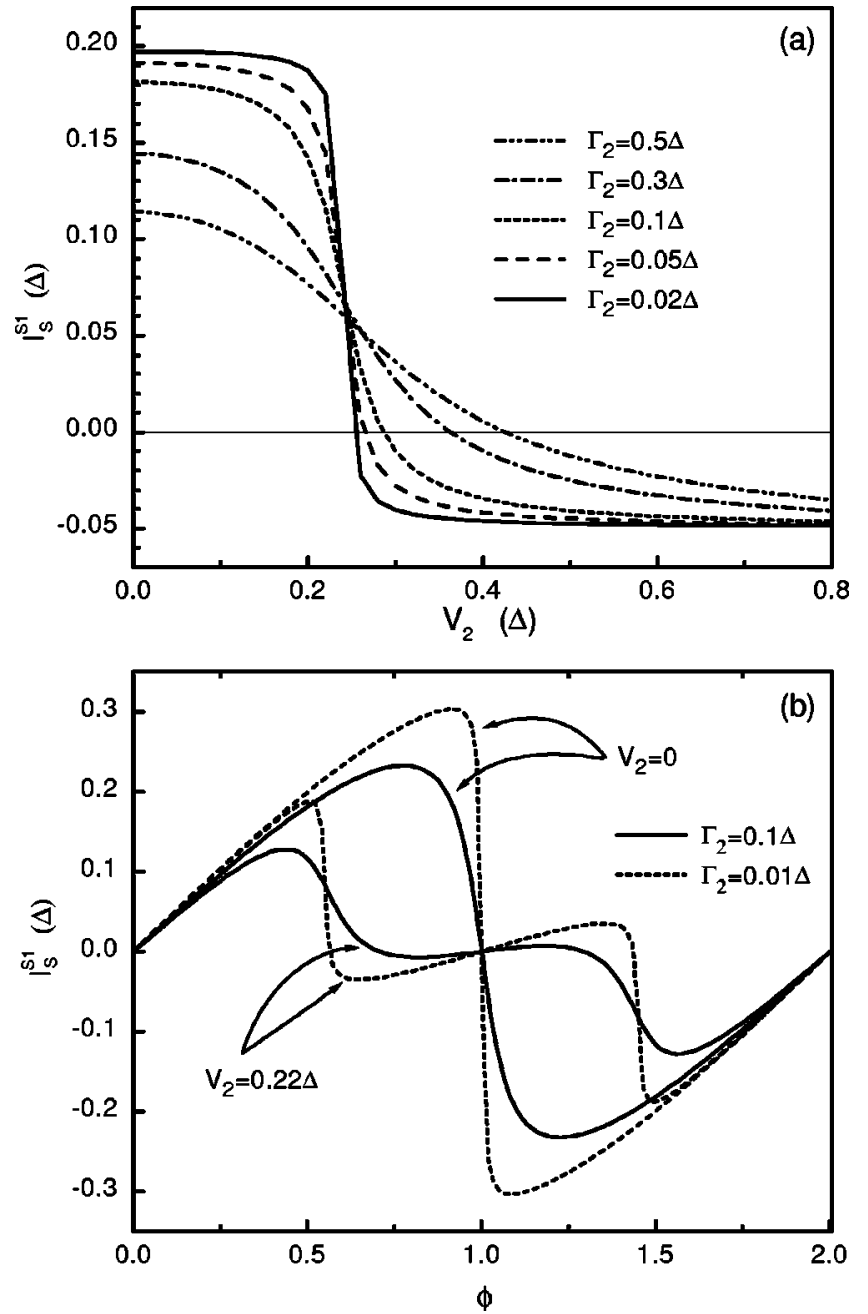

FIG. 10. $I_{S}^{S 1}$ in the limit of $\Gamma_{2} \rightarrow 0$. (a) $I_{S}^{S 1}$ vs $V_{2}$ with $\Gamma_{2}$ varying from $0.5 \Delta$, to $0.02 \Delta$. Other parameters are $\Gamma_{1}=\Gamma_{3}=0.5 \Delta$ and $\phi$ $=\pi / 2$. (b) $I_{S}^{S 1}$ vs $\phi$ at different $V_{2}$ and $\Gamma_{2}$, for $\Gamma_{1}=\Gamma_{3}=0.5 \Delta$.

to a two-terminal SNS junction. ${ }^{2}$ Then all other types of current approach zero, except the supercurrent $I_{S}^{S 1}$. The supercurrent $I_{S}^{S 1}$ vs $V_{2}$ is shown in Fig. 10(a). For small coupling strength $\Gamma_{2}$, a sudden change of $I_{S}^{S 1}$ occurs in the range of a few $\Gamma_{2}$ around $V_{2} \approx \epsilon_{A}^{+}$, due to the fact that the occupation number of the Andreev quasibound state varies from 0 to 1 while $V_{2}$ passes through $\epsilon_{A}^{+}$. $I_{S}^{S 1}$ vs $\phi$ at different $V_{2}$ is shown in Fig. 10(b), exhibiting also an abrupt change of $I_{S}^{S 1}$ vs $\phi$, while $\epsilon_{A}^{+}$, which also depends on $\phi$, passes through $V_{2}$. In particular, in the limit of $\Gamma_{2} \rightarrow 0$, the normal terminal $N 2$ tends to decouple from the system, but the supercurrent $I_{S}^{S 1}$ still depends on the bias $V_{2}$ of the normal terminal [see Figs. 10(a) and 10(b)]. The reason is that, when the density of states of quasiparticles in superconductors for $|\epsilon|<\Delta$ is zero, $\Sigma_{n}^{<}(\epsilon)=0 \quad(n=1,3)$ according to Eq. (26), and the occupation in the quantum dot in the gap region is completely determined by the bias of the normal terminal. Therefore the normal terminal $N 2$ can still affect the supercurrent even in the limit $\Gamma_{2} \rightarrow 0$. The same result was obtained previously by Wees et al. by using the scattering matrix method. ${ }^{2}$

It is interesting to notice that the supercurrent varies abruptly with the bias $V_{2}$ if the coupling strength $\Gamma_{2}$ is very small ( $\Gamma_{2}$ can not be zero) [see Fig. 10(a)]. This means that one can control the supercurrent $I_{S}^{S 1}$ by the bias $V_{2}$ very sensitively in the very weak coupling strength $\Gamma_{2}$. We expect that this behavior may be used to make a sensitive switch.

\section{CONCLUSIONS}

In this paper, we investigate the control of the supercurrent in a mesoscopic four-terminal SNS junction with a quantum dot as its $N$ region. By using the nonequilibrium Green's function method, the total terminal currents $\left(I^{S 1}\right.$ and $I^{N 2}$ ) and each type of the currents from the normal and superconducting terminal are derived and studied in detail. The supercurrent can be suppressed and the junction can be transformed into a $\pi$ junction, in agreement with recent experiments $^{1}$ and previous theories. ${ }^{10}$ We find that the current-phase relation of the conventional current between two normal terminals can be approximated as $I^{N 2}(\phi)$ $=I^{N 2}(0)(1+\alpha \cos \phi) /(1+\alpha)$ with $|\alpha|<1$. For the threeterminal SNS junction, even when all voltages of three terminals are set to be equal (under this condition no current is flowing between the dot and the normal terminal), the control of the supercurrent is still possible by tuning the coupling strength between the dot and the normal terminal $\Gamma_{2}$. For the case $V_{2} \neq 0$, we investigated the Andreev reflection current and the Andreev quasibound states in detail. Finally, the limit case of $\Gamma_{2} \rightarrow 0$ was studied, in which the supercurrent can also be controlled by changing the bias $V_{2}$, even in very small $\Gamma_{2}$.

We hope that the theoretical predictions of this work can be tested experimentally. As mentioned at the end of Sec. II, the experimental conditions for manifesting the control of the supercurrent in the setup under consideration, a fourterminal Josephson junction with a quantum dot in central region, is not harsh. A crucial condition for a clear manifestation of the effect is to keep only one of the energy levels of the quantum dot (usually a quantum dot has multiple energy levels $\left.\epsilon_{i}, i=0,1, \ldots\right)$, say $\epsilon_{0}$, be involved. This can be realized by (1) the bias voltages between the two normal leads and between the normal lead and the superconducting lead are small such that the maximum value $\max \left(\mid V_{2}\right.$ $\left.-V_{4}|,| V_{2}|,| V_{4} \mid\right)$ is less than the interval of the intradot Coulomb blockade oscillations $\delta \epsilon+U(\delta \epsilon$ is the intradot level spacing and $U$ is the intradot Coulomb interaction), then only the single particle energy levels need to be considered. (2) If only one level $\epsilon_{0}$ satisfies $\epsilon_{0}=0$, meanwhile no two levels within the gap region satisfy $\epsilon_{i}+\epsilon_{j}=0$, then only the level $\epsilon_{0}$ contributes to the supercurrent and the Andreev reflection current. ${ }^{14}$ However, if there are two levels satisfying $\epsilon_{i}+\epsilon_{j}$ $=0$, and with a larger bias voltage $\left|V_{2}-V_{4}\right|$, the Andreev reflection current will exist with more complicated pattern, but the basic features of the supercurrent, such as the reduction of $I_{S}^{S 1}$ vs $V_{2}$ in the four-terminal system and $I_{S}^{S 1}$ vs $\Gamma_{2}$ in the three-terminal system, will still maintain qualitatively.

\section{ACKNOWLEDGMENTS}

We gratefully acknowledge the financial support by a RGC grant from the SAR Government of Hong Kong under Grant No. HKU 7215/99P, a CRCG grant from the University of Hong Kong. T. H. Lin and Q. F. Sun were also sup- 
ported by the research grant from the Chinese National Natural Science Foundation, and the State Key Laboratory for Mesoscopic Physics in Peking University. The authors would like to thank the Computer Center of the University of Hong Kong for computational facilities. Q.F.S. and T.H.L. would like to thank B. G. Wang, X. A. Zhao, and Y. D. Wei for many helpful discussions.

\section{APPENDIX}

In this appendix, we present a simple derivation of the current $I^{N 2}$, flowing into the dot from the normal terminal N2. ${ }^{24}$ From Dyson's equation, one has

$$
G_{11}^{r *}\left(g_{11}^{r-1}-\Sigma_{11}^{r}\right) *-G_{12}^{r *} \Sigma_{21}^{r *}=1
$$

where $G_{11}^{r *}$ is $\left(G_{11}^{r}\right)^{*}$ and $g_{11}^{r-1}$ is $\left(g_{11}^{r}\right)^{-1}$. Multiplying $G_{11}^{r}$ on both sides of the Eq. (A1), one obtains

$$
G_{11}^{r}=\left|G_{11}^{r}\right|^{2}\left(g_{11}^{r-1}-\Sigma_{11}^{r}\right) *-G_{11}^{r} G_{12}^{r *} \Sigma_{21}^{r *},
$$

substituting $G_{11}^{r}$ into the current formula, Eq. (15), then we can rewrite the current $I^{N 2}$ as

$$
\begin{aligned}
I^{N 2}= & -4 e \operatorname{Im} \int \frac{d \epsilon}{2 \pi} \Gamma_{2} \\
& \times\left[f_{2}\left|G_{11}^{r}\right|^{2}\left(g_{11}^{r-1}-\Sigma_{11}^{r}\right) *-f_{2} G_{11}^{r} G_{12}^{r *} \Sigma_{21}^{r *}+\frac{1}{2} G_{11}^{<}\right] .
\end{aligned}
$$

It turns out that the conventional current $I_{c}^{N 2}$ is the combination of $f_{2}\left|G_{11}^{r}\right|^{2}\left(g_{11}^{r-1}-\Sigma_{11}^{r}\right) *$ and the first part of the Green function $G_{11}^{<}$[Eq. (28)] in Eq. (A3), and can be expressed as

$$
\begin{aligned}
I_{c}^{N 2}= & -4 e \operatorname{Im} \int \frac{d \epsilon}{2 \pi} \Gamma_{2}\left[f_{2}\left|G_{11}^{r}\right|^{2}\left(g_{11}^{r-1}-\Sigma_{11}^{r}\right) *\right. \\
& \left.+\frac{i}{2}\left|G_{11}^{r}\right|^{2}\left(\Gamma_{1} \tilde{\rho} f_{1}+\Gamma_{3} \tilde{\rho} f_{3}+\Gamma_{2} f_{2}+\Gamma_{4} f_{4}\right)\right] \\
= & 2 e \int \frac{d \epsilon}{2 \pi} \Gamma_{2}\left|G_{11}^{r}\right|^{2}\left[\Gamma_{1} \tilde{\rho}\left(f_{2}-f_{1}\right)\right. \\
& \left.+\Gamma_{3} \tilde{\rho}\left(f_{2}-f_{3}\right)+\Gamma_{4}\left(f_{2}-f_{4}\right)\right] .
\end{aligned}
$$

The rest part of the current $I^{N 2}$ is

$$
\begin{aligned}
& -2 e \operatorname{Im} \int \frac{d \epsilon}{2 \pi} \Gamma_{2}\left\{-2 f_{2} G_{11}^{r} G_{12}^{r *} \Sigma_{21}^{r *}\right. \\
& -i G_{11}^{r} G_{12}^{r * \frac{\Delta}{\epsilon}}\left(\Gamma_{1} \tilde{\rho} f_{1} e^{-i \phi_{1}}+\Gamma_{3} \tilde{\rho}_{3} e^{-i \phi_{3}}\right) \\
& -i G_{11}^{r *} G_{12}^{r} \frac{\Delta}{\epsilon}\left(\Gamma_{1} \tilde{\rho} f_{1} e^{i \phi_{1}}+\Gamma_{3} \tilde{\rho}_{3} e^{i \phi_{3}}\right) \\
& \left.+i\left|G_{12}^{r}\right|^{2}\left(\Gamma_{1} \tilde{\rho} f_{1}+\Gamma_{3} \tilde{\rho} f_{3}+\Gamma_{2} \widetilde{f}_{2}+\Gamma_{4} \widetilde{f}_{4}\right)\right\} .
\end{aligned}
$$

Again by using Eq. (21), the first term in Eq. (A5) can be rewritten as

$$
-2 f_{2} G_{11}^{r} G_{12}^{r *} \Sigma_{21}^{r *}=-2 f_{2}|A|^{2}\left(g_{22}^{r-1}-\Sigma_{22}^{r}\right) \Sigma_{12}^{r *} \Sigma_{21}^{r *} .
$$

Furthermore, starting with

$$
\begin{aligned}
\Sigma_{21}^{r *}= & \frac{-i \Delta}{2 \epsilon} \beta^{*}(\epsilon)\left(\Gamma_{1} e^{-i \phi_{1}}+\Gamma_{3} e^{-i \phi_{3}}\right)=\Sigma_{12}^{r} \theta(\Delta-|\epsilon|) \\
& -\Sigma_{12}^{r} \theta(|\epsilon|-\Delta) \\
= & \Sigma_{12}^{r}-2 \Sigma_{12}^{r} \theta(|\epsilon|-\Delta)
\end{aligned}
$$

the rest part of $I^{N 2}$ can be rewritten in the form

$$
\begin{aligned}
& -2 e \operatorname{Im} \int \frac{d \epsilon}{2 \pi} \Gamma_{2}\left\{-2 f_{2}\left|G_{12}^{r}\right|^{2}\left(g_{22}^{r-1}-\Sigma_{22}^{r}\right)\right. \\
& +4 f_{2}\left|G_{12}^{r}\right|^{2}\left(g_{22}^{r-1}-\Sigma_{22}^{r}\right) \theta(|\epsilon|-\Delta) \\
& +2 i \operatorname{Re}\left[-G_{11}^{r} G_{12}^{r *} \frac{\Delta}{\epsilon}\right. \\
& \times\left(\Gamma_{1} \tilde{\rho} f_{1} e^{\left.\left.-i \phi_{1}+\Gamma_{3} \tilde{\rho} f_{3} e^{-i \phi_{3}}\right)\right]}\right. \\
& \left.+i\left|G_{12}^{r}\right|^{2}\left(\Gamma_{1} \tilde{\rho} f_{1}+\Gamma_{3} \tilde{\rho} f_{3}+\Gamma_{2} \tilde{f}_{2}+\Gamma_{4} \tilde{f}_{4}\right)\right\} .
\end{aligned}
$$

By combining the first and fourth terms in Eq. (A8), the Andreev current $I_{A}^{N 2}$ and $I_{1}^{N 2}$ can be expressed, respectively, as

$$
\begin{gathered}
I_{A}^{N 2}=2 e \int \frac{d \epsilon}{2 \pi} \Gamma_{2}\left|G_{12}^{r}\right|^{2}\left[\Gamma_{2}\left(f_{2}-\widetilde{f}_{2}\right)+\Gamma_{4}\left(f_{2}-\widetilde{f}_{4}\right)\right], \\
I_{1}^{N 2}=2 e \int \frac{d \epsilon}{2 \pi} \Gamma_{2}\left|G_{12}^{r}\right|^{2}\left[\Gamma_{1} \tilde{\rho}\left(f_{2}-f_{1}\right)+\Gamma_{3} \tilde{\rho}\left(f_{2}-f_{3}\right)\right] .
\end{gathered}
$$

Finally, it is easy to see that the second and third terms in Eq. (A8), i.e., 


$$
\begin{aligned}
& -2 e \operatorname{Im} \int \frac{d \epsilon}{2 \pi} \Gamma_{2}\left\{4 f_{2}\left|G_{12}^{r}\right|^{2}\left(g_{22}^{r-1}-\Sigma_{22}^{r}\right) \theta(|\epsilon|-\Delta)\right. \\
& \left.+2 i \operatorname{Re}\left[G_{11}^{r} G_{12}^{r *} \frac{-\Delta}{\epsilon}\left(\Gamma_{1} \tilde{\rho} f_{1} e^{-i \phi_{1}}+\Gamma_{3} \tilde{\rho} f_{3} e^{-i \phi_{3}}\right)\right]\right\},
\end{aligned}
$$

constitute the current $I_{2}^{N 2}$, just by noticing that

$$
\begin{aligned}
\operatorname{Im}\left[4 f_{2}\left|G_{12}^{r}\right|^{2}(\right. & \left.\left.g_{22}^{r-1}-\Sigma_{22}^{r}\right) \theta(|\epsilon|-\Delta)\right] \\
= & \operatorname{Im}\left[4 f_{2} G_{11}^{r} G_{12}^{r *} \Sigma_{12}^{r} \theta(|\epsilon|-\Delta)\right] \\
=\operatorname{Im} & {\left[4 f_{2} G_{11}^{r} G_{12}^{r *} \frac{i \Delta}{2 \epsilon} \beta(\epsilon)\right.} \\
& \left.\times\left(\Gamma_{1} e^{-i \phi_{1}}+\Gamma_{3} e^{-i \phi_{3}}\right) \theta(|\epsilon|-\Delta)\right] \\
= & \operatorname{Im}\left[2 f_{2} G_{11}^{r} G_{12}^{r *} \frac{i \Delta}{\epsilon} \widetilde{\rho}\left(\Gamma_{1} e^{-i \phi_{1}}+\Gamma_{3} e^{-i \phi_{3}}\right)\right],
\end{aligned}
$$

which can be expressed as

$$
\begin{aligned}
I_{2}^{N 2}= & -2 e \int \frac{d \epsilon}{2 \pi} \Gamma_{2} \frac{\tilde{\rho}^{2 \Delta}}{\epsilon} \operatorname{Re}\left\{G_{11}^{r} G_{12}^{r *} e^{-i \phi_{1}} \Gamma_{1}\left(f_{2}-f_{1}\right)\right. \\
& \left.+G_{11}^{r} G_{12}^{r *} e^{-i \phi_{3}} \Gamma_{3}\left(f_{2}-f_{3}\right)\right\} .
\end{aligned}
$$

Therefore the current $I^{N 2}$ flowing into the dot from the normal terminal $N 2$ can be expressed as Eqs. (32) and (33). Similarly, one can also calculate the current $I^{S 1}$. It is worth mentioning that only the total current $I^{N 2}$ (or $I^{S 1}$ ) flowing from one terminal can be measured. It is impossible to distinguish different types of current in the measurement. That is why we mainly focus on the investigation of the total currents $I^{S 1}$ and $I^{N 2}$ in the text; only in Sec. IV(B) we presented the behavior of each type of currents in Fig. 8. (Note that for many cases discussed in the text, only one type of the current, supercurrent or Andreev current, is nonzero.)
${ }^{1}$ J.J.A. Baselmans, A.F. Morpurgo, B.J. van Wees, and T.M. Klapwijk, Nature (London) 397, 43 (1999).

${ }^{2}$ B.J. van Wees, K.-M.H. Lenssen, and C.J.P.M. Harmans, Phys. Rev. B 44, 470 (1991).

${ }^{3}$ G. Wendin and V.S. Shumeiko, Phys. Rev. B 53, R6006 (1996).

${ }^{4}$ L.-F. Chang and P.F. Bagwell, Phys. Rev. B 55, 12678 (1997).

${ }^{5}$ A.F. Volkov, Phys. Rev. Lett. 74, 4730 (1995).

${ }^{6}$ A.F. Volkov and H. Takayanagi, Phys. Rev. B 56, 11184 (1997).

${ }^{7}$ S.-K. Yip, Phys. Rev. B 58, 5803 (1998).

${ }^{8}$ P.F. Bagwell, Phys. Rev. B 46, 12573 (1992).

${ }^{9}$ A.F. Morpurgo, T.M. Klapwijk, and B.J. van Wees, Appl. Phys. Lett. 72, 966 (1998).

${ }^{10}$ F.K. Wilhelm, G. Schön, and A.D. Zaikin, Phys. Rev. Lett. 81, 1682 (1998).

${ }^{11}$ H. Pothier, S. Gueron, Norman O. Birge, D. Esteve, and M. H. Devoret, Phys. Rev. Lett. 79, 3490 (1997).

${ }^{12}$ J.C. Cuevas, A. Martin-Rodero, and A. Levy Yeyati, Phys. Rev. B 54, 7366 (1996).

${ }^{13}$ S. Ishizaka, J. Sone, and T. Ando, Phys. Rev. B 52, 8358 (1995).

${ }^{14}$ Q.-F. Sun, J. Wang, and T.-H. Lin, Phys. Rev. B 59, 3831 (1999).

${ }^{15}$ To compare with Refs. 12 and 13, the Hamiltonian of the superconducting leads have a difference in the pairing potential term: $\Sigma_{k} \Delta a_{k \uparrow} a_{-k \downarrow}$ in their paper and $\Sigma_{k} \Delta a_{k \downarrow} a_{-k \uparrow}$ here. This difference is equivalent to add a $\pi$ to the superconducting phase in ALL superconductor (due to $\Sigma_{k} \Delta a_{k \downarrow} a_{-k \uparrow}=-\Sigma_{k} \Delta a_{-k \uparrow} a_{k \downarrow}$ $\left.=\Sigma_{k} \Delta e^{i \pi} a_{k \uparrow} a_{-k \downarrow}\right)$, therefore this difference does not affect any result.

${ }^{16}$ B.J. Van Wees and H. Takayanagi, in Mesoscopic Electron Transport, Vol. 345 of NATO Advanced Studies Institute Series $E$, edited by L.L. Sohn et al. (Kluwer Academic, Dordrecht, 1997), pp. 469-502.

${ }^{17}$ Q.-F. Sun, B.-G. Wang, J. Wang, and T.-H. Lin, Phys. Rev. B 61, 4754 (2000).

${ }^{18}$ N.S. Wingreen, Antti-Pekka Jauho, and Y. Meir, Phys. Rev. B 48, 8487 (1993).
${ }^{19}$ Antti-Pekka Jauho, N.S. Wingreen, and Y. Meir, Phys. Rev. B 50, 5528 (1994).

${ }^{20}$ A. Martin-Rodero, F.J. Garcia-Vidal, and A.L. Yeyati, Phys. Rev. Lett. 72, 554 (1994).

${ }^{21}$ Q.-F. Sun, J. Wang, and T.-H. Lin, Phys. Rev. B 59, 13126 (1999).

${ }^{22}$ N.S. Wingreen, K.W. Jacobsen, and J.W. Wilkins, Phys. Rev. B 40, 11834 (1989).

${ }^{23}$ Q.-F. Sun and T.-H. Lin, J. Phys.: Condens. Matter 9, 3043 (1997); 9, 4875 (1997).

${ }^{24}$ From the equation of $2 i \operatorname{Im} \mathbf{G}^{r}=\mathbf{G}^{r}-\mathbf{G}^{a}=\mathbf{G}^{r}\left(\boldsymbol{\Sigma}^{r}-\mathbf{\Sigma}^{a}\right) \mathbf{G}^{a}$, one can also derive the current formulas $I^{N 2}$ and $I^{S 1}$, i.e., Eqs. (30)(33).

${ }^{25}$ Q.-F. Sun, J. Wang, and T.-H. Lin, Phys. Rev. B 58, 13007 (1998).

${ }^{26}$ Y. Meir, N.S. Wingreen, and Patrick A. Lee, Phys. Rev. Lett. 66, 3048 (1991).

${ }^{27}$ Z.S. Ma, J. Wang, and H. Guo, Phys. Rev. B 57, 9108 (1998); 59, 7575 (1999).

${ }^{28}$ In fact, here $j(\epsilon)$ multiplied by a factor of $2\left[\left(\Gamma_{1} / \Gamma_{2}\right) \tilde{\rho}+1\right]$ is similar to the functions $I_{n}^{ \pm}(\phi)$ and $I(E, \phi)$ in Ref. 8. And they reflect the supercurrent carrying per unit energy by the intradot electron.

${ }^{29}$ H. Nakano and H. Takayanagi, Phys. Rev. B 47, 7986 (1993); Solid State Commun. 80, 997 (1991).

${ }^{30}$ S.G. den Hartog, C. M. A. Kapteyn, B. J. van Wees, and T. M. Klapwijk, Phys. Rev. Lett. 77, 4954 (1996).

${ }^{31}$ H.T. Ilhan, H.V. Demir, and P.F. Bagwell, Phys. Rev. B 58, 15 120 (1998).

${ }^{32}$ T. Akazaki, H. Takayanagi, J. Nitta, and T. Enoki, Appl. Phys. Lett. 68, 418 (1996).

${ }^{33}$ Due to the coupling between the dot and the normal lead, the Andreev bound states are quasibound states with a certain spreading of the energy levels. 